\title{
The beholder's share in the perception of orientation of 2-D shapes
}

\author{
BAOXIA LIU, TJEERD M. H. DIJKSTRA, and AUGUSTINUS H. J. OOMES \\ Ohio State University, Columbus, Ohio
}

\begin{abstract}
A considerable amount of research demonstrates that people perceive cardinal orientations (horizontal and vertical) more accurately than other orientations; this is termed the oblique effect. We investigated the interaction of this effect with the degree of elongation of the stimulus. Our stimuli were ellipses with a wide range of aspect ratios, varying from a circle (aspect ratio $=1$ ) to a line (aspect ratio = 123.5). The task was to set a probe line in the same orientation as the long axis of the ellipse. In our first experiment, we determined that performance is degraded as the aspect ratio decreases; furthermore, the bias and response variability are linearly related to a transformation of aspect ratio (round$n e s s$ ). We found significant individual differences; the results show high within-subjects correlations and low between-subjects correlations. In our second experiment, we had observers judge the orientation of circles randomly mixed in with ellipses of low aspect ratio. The observers demonstrated intrinsic preferences and generated reproducible distributions of orientation settings with idiosyncratic profiles. These distributions predict the influence on the response to ellipses with an aspect ratio higher than one and can be considered as the beholder's share in the perception of shape orientation.
\end{abstract}

Previous research on orientation perception of twodimensional (2-D) shapes has demonstrated a higher performance at cardinal orientations (horizontal and vertical) than at oblique orientations. This superiority of performance around the vertical and the horizontal is called the oblique effect (see the reviews of Appelle, 1972; Howard, 1982; Regan, 2000). Two kinds of stimuli are usually used: gratings and lines. Research with gratings has shown that there exist gratings with relatively high spatial frequencies or low contrast that cannot be detected at oblique orientations but are easily resolvable at cardinal orientations (Bowker \& Mandler, 1981). In the research on the orientation perception of lines, observers are usually asked to match or discriminate line orientations. The results are reported as the accuracy and the precision of the judgments. Accuracy is quantified by the difference between the stimulus orientation and the mean of the probe orientations as set by an observer or the discrimination threshold of noticing an orientation difference between two stimuli. Low values indicate a small bias and a high accuracy. Precision is quantified by the standard deviation of the responses around the mean. A small standard deviation refers to a highly concentrated distribution and a high precision.

The oblique effect results in higher accuracy and precision in the ability to match and discriminate orienta-

This work was supported by National Science Foundation Grant SBR9809447 and by a seed grant from The Ohio State University. We thank Manoj Subbaram and Mark A. Bullimore of the College of Optometry at OSU for testing our observers for astigmatism. We thank Jim Crowell, Jim Todd, and two anonymous reviewers for helpful suggestions. Correspondence concerning this article should be addressed to B. Liu, School of Optometry, University of California, 360 Minor Hall, Berkeley, CA 94720 (e-mail: baoxia@uclink.berkeley.edu). tions around the vertical and the horizontal than in other orientations, and many empirical results show this effect. Andrews (1967) presented a short line at the fovea and asked observers to set it parallel to a long reference line. The results showed the oblique effect both in accuracy and in precision. Bouma and Andriessen (1968) asked observers to set a point on the extension of a line segment and obtained similar results; the point setting was more accurate and precise for cardinal orientations than for oblique ones. Westheimer and Beard (1998) used an orientation discrimination task to investigate the orientation perception of lines. Observers were shown a line at a specific orientation, followed by another line at another orientation, and they were asked whether the second one was clockwise or counterclockwise relative to the orientation of the first line. Results of this orientation discrimination task again showed the oblique effect.

There is much discussion about the origin of the oblique effect. Two types of explanations have been put forward: those based on properties of neural processing (see Howard, 1982, for a review) and those that seek the origin of the oblique effect in the prevalence of contours with cardinal orientations in the environment (Coppola, Purves, McCoy, \& Purves, 1998; Switkes, Mayer, \& Sloan, 1978). Recent research has demonstrated that higher level processing contributes to the oblique effect. First, $\mathrm{Li}$ and Westheimer (1997) reported an oblique effect for the overall orientation of a configuration of elements. $\mathrm{Li}$ and Westheimer used two intersecting line elements as stimuli. The lines were at an oblique angle with each other, like a squashed "x." Thus, the orientation of the line elements was different from the orientation of the whole configuration; they termed the latter the implicit orienta- 
tion. This raises the question of which orientation mediates the oblique effect: the orientation of the line elements or that of the whole configuration. In their experiments, observers were asked to indicate whether a stimulus (the whole configuration) was clockwise or counterclockwise to vertical. Results indicated that the oblique effect was determined by the implicit orientation. A second contribution of higher level processing was demonstrated by Vogels and Orban (1985), who showed that practice improves performance for oblique orientations more than for the principal ones. In addition, Heeley and BuchananSmith (1990) showed that observers might depend on their memory or labeling of vertical and horizontal to assist their judgment of orientation. Two tasks were used in their experiments: In the one-interval task, one stimulus was presented, and observers indicated whether it was tilted clockwise or counterclockwise to vertical or horizontal; in the two-interval task, two stimuli were presented (one of the stimuli was always vertical or horizontal), and observers were asked which one of them was more tilted than the other one in a clockwise direction. Observers' performance was similar under the two tasks, which showed that they might use their memory or labeling of the cardinal orientations to assist their orientation discrimination.

For some simple shapes, such as the ellipse, the orientation of the whole shape is not the same as the orientation of its boundary, so research on this kind of shapes provides more insights about the origin of the oblique effect. Previous experiments on simple shapes showed that aspect ratio (the ratio between the lengths of the long axis and the short axis of a shape) is closely related to the performance of orientation perception. In one of their experiments, Li and Westheimer (1997) showed that the precision with which the orientation of the long axis of an ellipse was discriminated increased with aspect ratio. Ellipses with higher aspect ratio are more like an oval and less like a circle, so the increase in precision is not unexpected. In an experiment on three-dimensional (3-D) orientation perception, Oomes and Dijkstra (2002) found a similar advantage for more elongated shapes. In their study, it was realized that orientation perception of 3 -D shapes (slant and tilt) from binocular information should be dependent on how accurate and precise the orientation of each contour was in each eye. However, there exists no comprehensive data set on the accuracy and precision of perception of 2-D orientation in the literature. Thus, the first purpose of the present study was to quantify both accuracy and precision of perception of orientation of 2-D shapes in enough detail to be used for the purpose of building a model of shape from binocular contour information. Thus, we used ellipses with a large range of aspect ratios and orientations. In collecting the present data set, we realized that many of the effects of orientation on accuracy and precision could be modeled in a Bayesian framework. Thus, a second purpose was the description of a set of data that could support a Bayesian model.

\section{EXPERIMENTS}

We performed three experiments. Experiment 1 was designed to explore the orientation perception of ellipses over a wide range of conditions. In Experiment 2, we measured the observers' intrinsic preference in the absence of any orientation information and used that datum to model certain aspects of the data. In Experiment 3, we used irregular shapes that were generated by randomly perturbing the contour of ellipses to establish whether the results from the first two experiments would hold up for more generic shapes. The observers, apparatus, probe, and procedure were the same for all three experiments and will be described in the next section.

\section{General Method}

Subjects. Six observers ( 3 males, 3 females) participated in the experiment; their ages ranged from 25 to 29 years. We will call them A, B, C, S, V, and Y hereafter. They all had normal or corrected-tonormal visual acuity. Since some of our results might be explained by uncorrected astigmatism, we had our observers checked for astigmatism by an objective procedure. Astigmatism was checked with a Humphrey Automatic Refractor Keratometer, Model 599. We found that 4 observers had no significant astigmatism in either eye-that is, less than 0.5 diopter. Observer A had astigmatism of 0.62 diopter in his left eye $\left(19^{\circ}\right.$ counterclockwise from the horizontal) and no signif icant astigmatism in his right eye. Observer $\mathrm{C}$ had astigmatism of 0.75 diopter in his left eye $\left(23^{\circ}\right.$ counterclockwise from the horizontal) and no significant astigmatism in his right eye. None of the observers had experience with psychophysical experiments, and they were unaware of the purpose of the experiments. Five of the observers received payment for participation. All the observers provided consent in accordance with the Ohio State University Institutional Review Board.

Apparatus. The experiments were run on a Silicon Graphics Indigo $^{2}$ with OpenGL graphics package for the production and the presentation of the stimuli. The monitor was a Sony GDM-20D11 19-in. CRT with a frame rate of $72 \mathrm{~Hz}$. The stimuli were presented within a $35.0 \mathrm{~cm}$ (horizontal) $\times 28.0 \mathrm{~cm}$ (vertical) rectangular region of the display screen with a spatial resolution of $1,280 \times 1,024$ pixels. The displays were viewed binocularly from a distance of $114 \mathrm{~cm}$, so that the screen subtended $17.5^{\circ} \times 14.0^{\circ}$ of visual angle and each pixel subtended $0.82 \mathrm{~min}$. The observer's head was stabilized with a chinrest. There were dim lights above the monitor.

Stimuli. The stimuli were ellipses with different aspect ratios and orientations. The aspect ratio is the ratio of the length of an ellipse's long (major) axis to that of its short (minor) axis. A circle and a line are extreme cases of an ellipse: An ellipse with aspect ratio of 1 is a circle, and an ideal line can be thought of as an ellipse with an infinite aspect ratio. All the stimuli were presented at the center of the screen as solid white shapes on gray backgrounds (see Figure 1). The stimuli were drawn as polygons with 120 sides. This provided close approximations to ellipses.

To make sure that the boundary of the ellipse was devoid of jaggies, the ellipses were made as large as possible. However, the size of the monitor screen limited the length of the major axis. The size of the ellipses was generated with both of these considerations in mind. For aspect ratios less than 2.0, the area of the ellipse ( $\pi$ multiplied by the lengths of the long and short axes) was kept constant at $72.35 \mathrm{~cm}^{2}$. The length of the major axis for ellipses with aspect ratios larger than or equal to 2.0 was kept constant at $13.58 \mathrm{~cm}$. The length of the short axis was calculated according to its aspect ratio. We also used a line segment with an aspect ratio of 123.5; it mea- 


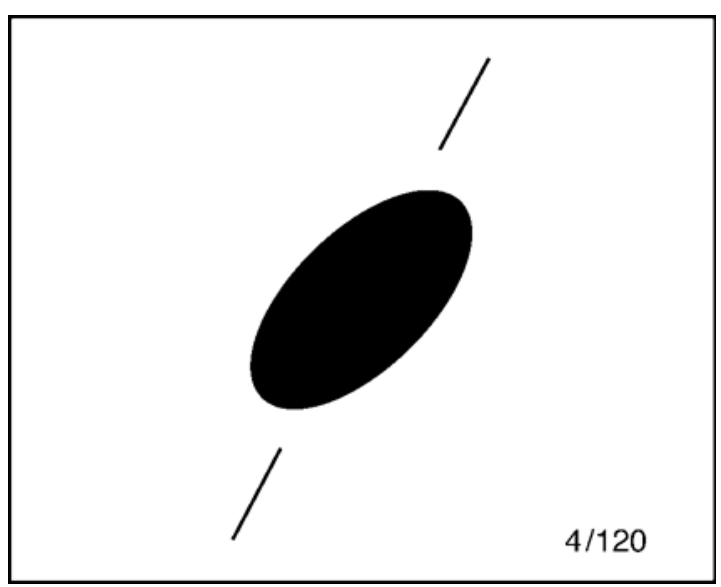

Figure 1. An illustration of the stimulus display in Experiment 1 and Experiment 2. In the experiments, the ellipse and the line segments were presented as white, and the background was gray. The ellipse in the figure has an aspect ratio of 2.0 and an orientation of $45^{\circ}$. The probe line is set counterclock wise relative to the long axis of the ellipse and has a positive bias. The two numbers at the bottom right are the current trial number and the total number of trials in the block, and they were shown as white in the experiments.

sured $13.58 \mathrm{~cm}$ long $\left(6.82^{\circ}\right.$ of visual angle $)$ by $0.11 \mathrm{~cm}$ wide. A discussion of spatial accuracy of stimulus and probe placement is provided in Appendix A.

Probe. The probe consisted of two collinear line segments, one on either side of the stimulus and symmetrically placed around the screen center (Figure 1). The distance between the outer endpoints of the segments was $27.0 \mathrm{~cm}$ in all the conditions. The orientation of the probe line could be changed by dragging the mouse. The minimum distance from the inner ends of the line segments to the stimulus (i.e., the distance when the line segments were aligned with the major axis) was kept constant at $1.5 \mathrm{~cm}$ in each condition. This meant that the length of the line segments and the size of the gap between the line segments varied with the aspect ratio of the ellipse. Within each trial, the probe line segments did not change their lengths when their orientations were changed. This was done in order to keep the position cue, where an observer minimizes the distance between ellipse and probe line, as constant as possible across conditions.

Procedure. A stimulus and a probe were presented on the screen, as is shown in Figure 1. The orientation of the probe line segments was randomly chosen at the beginning of each trial.

We had the observers estimate the orientation of the ellipse, using the method of adjustment. The task was to rotate the probe (the broken line) with the mouse to make its orientation appear the same as the orientation of the long axis of the ellipse. When satisfied with the setting, the observer ended a trial by pressing the space bar. The observers received no feedback about their performance.

\section{EXPERIMENT 1 Ellipse Orientation Perception}

In the first experiment, we investigated the observers' performance at judging ellipse orientation.

\section{Method}

Design. This experiment was a $9 \times 12$ within-subjects design. Aspect ratio and orientation were the two independent variables. The stimulus had one of nine different aspect ratios: 1.02, 1.05,
$1.10,1.20,1.50,2.0,8.0,16.0$, and 123.5 (line). The orientation of the stimulus was chosen from 12 orientations: $0^{\circ}, 15^{\circ}, 30^{\circ}, 45^{\circ}, 60^{\circ}$, $75^{\circ}, 90^{\circ}, 105^{\circ}, 120^{\circ}, 135^{\circ}, 150^{\circ}$, and $165^{\circ}$ (measured counterclockwise from the horizontal). See Figure 2 for a sample of the conditions.

Stimuli and Procedure. The stimulus was an ellipse or a line, as was described in the General Method section. Five of the 6 observers did 2 practice blocks, with all the combinations of the 9 aspect ratios and 12 orientations randomly presented in each block (108 trials in 1 block). In the 1st block, stimuli were presented as described in the General Method section. In the 2nd block, the ellipses were presented with their long axis marked as a red line, to ensure that the observers understood the task. Observer C did only the 2nd practice block, with the long axis of the ellipse marked as a red line. After practice, each observer did 40 blocks of experimental trials during a period of 2 weeks. In each block, each combination of 12 orientations and 9 aspect ratios (108 trials) was presented in random order. The time it took for an observer to finish 1 block was around half an hour. Each observer did 40 repeats for each condition, which was a total of 4,320 trials for 1 observer.

\section{Results}

We summarized the observers' performance in terms of the circular mean bias and the circular standard deviation (Fisher, 1993). The bias was calculated by subtracting the orientation of the stimulus from the mean orientation of the probe settings. Linear statistics is not applicable in our case because the standard deviation in some conditions exceeds $20^{\circ}$ and, in that case, circular statistics is the appropriate method (Fisher, 1993). The relevant formulas from circular statistics are listed in Appendix B.

We excluded some of the data on the basis of two considerations. First, we excluded individual trials (outliers) on the basis of the likelihood of the excluded trial, given the distribution of the other trials. Second, we excluded whole aspect ratio conditions from the regressions plotted in Figures 4 and 5. These exclusions were based on the intrinsic preferences of the observers measured in Experiment 2 and will be discussed in connection with Figure 12.

Outliers were detected as follows. The circular standard deviations were calculated for each aspect ratio and for each observer, averaged over orientation and trial. Only those aspect ratio conditions with circular standard de-

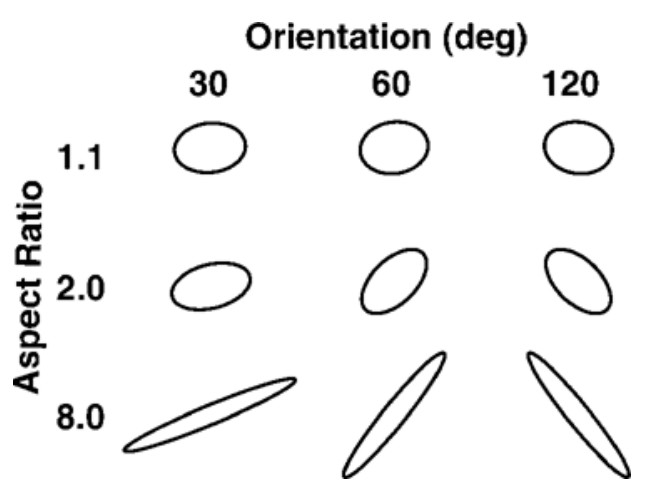

Figure 2. A sample of the conditions of Experiment 1. The different rows are different aspect ratios; the different columns are different orientations. 
viations smaller than $15^{\circ}$ were scrutinized for outliers. If the circular standard deviation was larger than $15^{\circ}$, we did not exclude any trials. Thus, for Subject A, only aspect ratios of 1.1 and above were scrutinized. For Subjects $\mathrm{B}, \mathrm{C}, \mathrm{S}, \mathrm{V}$, and $\mathrm{Y}$, these aspect ratios were 1.02, 1.1, $1.5,1.1$, and 1.05 , respectively. Individual trials were excluded on the basis of their deviation from the mean of the other settings for any given condition. The maximum deviation allowed was the same for all the subjects and all the orientations but varied with aspect ratio. The maximum deviation allowed was $90^{\circ}, 60^{\circ}, 45^{\circ}, 30^{\circ}, 22.5^{\circ}$, $18^{\circ}, 6^{\circ}, 6^{\circ}$, and $6^{\circ}$ for aspect ratios of $1.02,1.05,1.1,1.2$, $1.5,2,8,16$, and 123.5. In total, we excluded 35 trials, amounting to $0.14 \%$ of all the data $(25,920)$ from the analysis. In order to check that these data were outliers, we calculated their $z$-score on the basis of the circular standard error of the nonexcluded trials. We found a range of $z$-scores from 28 to 640, with an average of 140, underscoring the fact that the excluded trials are very unlikely to occur given the other trials. These large $z$-scores indicate why the exclusion was necessary: These trials would exert an undue influence on the regressions in Figures 4 and 5.
General results: Biases and circular standard deviations. We first will present a subset of the results for 1 observer. We will highlight the main findings and then will present more detailed analyses in separate sections. Observer A's bias and circular standard deviation as a function of ellipse orientation for three different aspect ratios $(1.05,1.50$, and 16.0) are shown in Figure 3. Plots of the observer's bias are shown in the top panels in the figure; note the change in vertical axis scale between plots. The sign of the bias indicates the direction of the setting, where positive means that the setting of the probe was counterclockwise from the orientation of the ellipse. The error bars in the plots are $95 \%$ confidence intervals (see Appendix B).

The first thing to note is that as the aspect ratio increases from left to right in the top row, the biases decrease. More precisely, the pattern of biases as a function of stimulus orientation roughly follows a sine wave pattern, and the amplitude of this sine wave decreases with aspect ratio. Second, note that for aspect ratios of 1.5 and 16 , the biases have zero crossings around the vertical and horizontal orientations; the fact that the biases are smallest in magnitude at these points is an aspect of the oblique
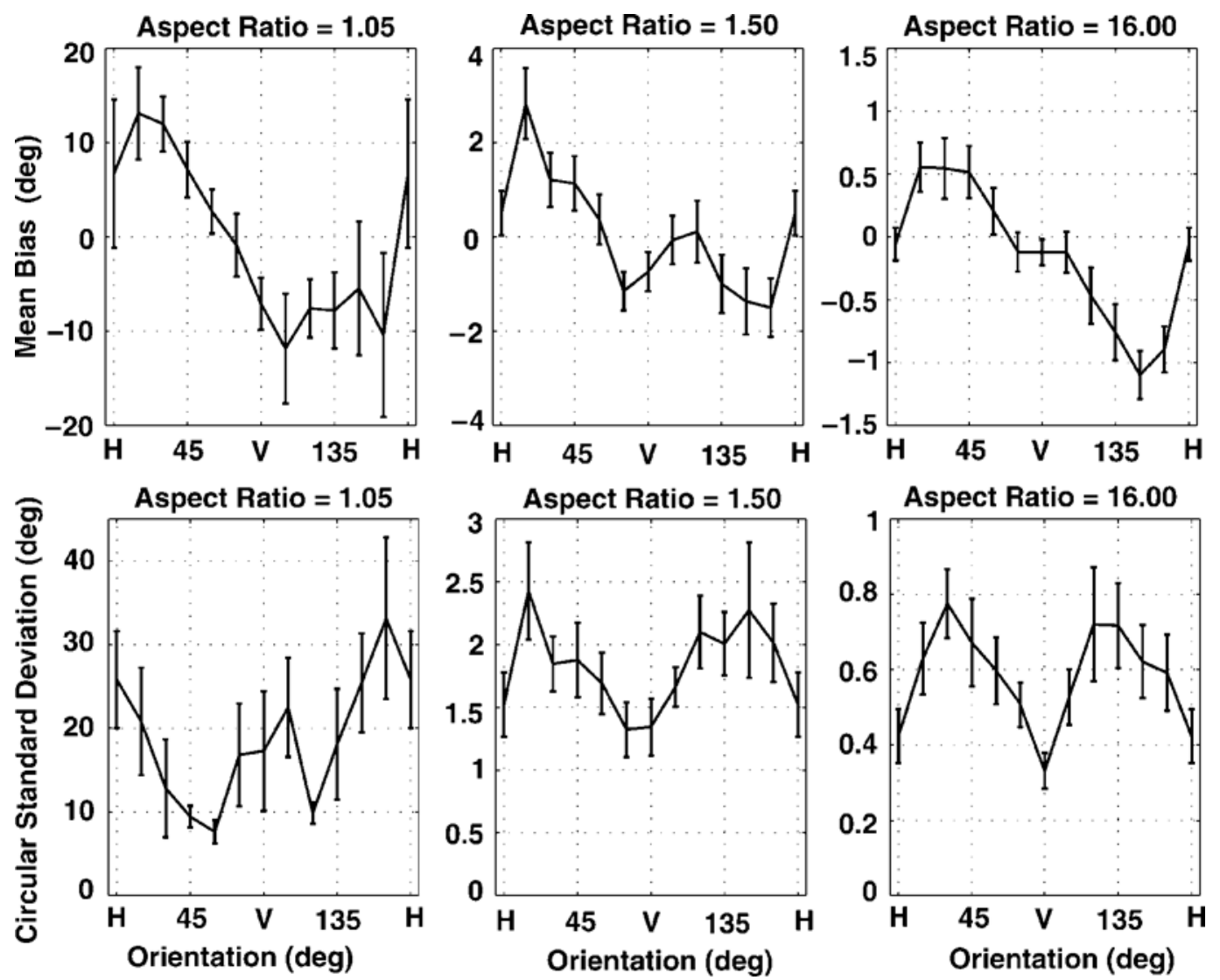

Figure 3. Observer A's results for aspect ratios of 1.05, 1.5, and 16. The top panels show the bias results; the bottom panels show the circular standard deviation results. The error bars denote the $95 \%$ confidence interval. " $H$ " and " $V$ " indicate horizontal and vertical orientations, respectively. 
effect. In more detail, the positive zero crossing around the horizontal $\left(0^{\circ}\right)$ is steep; the one around the vertical $\left(90^{\circ}\right)$ is shallow for an aspect ratio of 1.5 and is absent for an aspect ratio of 16 . The steep positive zero crossing near $0^{\circ}$ indicates that the responses tended to be biased away from the horizontal. To take a specific example, consider the $3^{\circ}$ bias for an ellipse orientation of $15^{\circ}$ counterclockwise and aspect ratio of 1.5. The subject judged the orientation of the ellipse to be $18^{\circ}$ counterclockwise, a repulsion from the horizontal. Similarly, we find a bias of $-1.5^{\circ}$ for an ellipse at an orientation of $165^{\circ}$ (i.e., $15^{\circ}$ clockwise). Thus, the subject judged the orientation of the ellipse to be $163.5^{\circ}$ (i.e., $16.5^{\circ}$ clockwise), illustrating again a repulsion. Similarly, a negative zero crossing represents an attraction to the orientation at the zero crossing. All the observers' data had positive crossings close to the vertical or the horizontal. Most showed more robust crossings than did Observer A. There was no clear pattern for the negative zero crossings. Also discussed in connection with Figure 11 will be the finding that the negative zero crossings of bias show no clear pattern for the higher aspect ratios. As is shown in Figure 1, Observer A's bias for an aspect ratio of 1.05 has a zero crossing around $60^{\circ}$ (negative) and $170^{\circ}$ (positive). The pattern of zero crossings for this aspect ratio condition tended to be quite idiosyncratic: Each observer has his or her own zero crossings. The negative zero crossings for other observers are around $65^{\circ}(\mathrm{B}), 125^{\circ}(\mathrm{C}), 90^{\circ}(\mathrm{S}), 55^{\circ}$ and $145^{\circ}(\mathrm{V})$, and $90^{\circ}(\mathrm{Y})$. An explanation for this finding will be provided in connection with Figure 11.

Observer A's circular standard deviations for the same three aspect ratios are plotted in the bottom panels in Figure 3 . We note that, like the bias, the circular standard deviations also decreased with increasing aspect ratio. Furthermore, the circular standard deviation for aspect ratios of 1.5 and 16.0 was smaller around the vertical and the horizontal than at other orientations; this is another aspect of the oblique effect. The other observers showed the same pattern. Aspect ratio 1.05 seems to be exceptional here, in that the circular standard deviation has a broad minimum around $60^{\circ}$. As for the bias pattern, the pattern of minima for this aspect ratio condition tended to be quite idiosyncratic for different observers. Each observer's data exhibited one or two minima. The minima for the aspect ratio of 1.05 for the other 5 observers, whose data are not shown, were approximately $0^{\circ}$ and $90^{\circ}(\mathrm{B}), 60^{\circ}$ and $130^{\circ}(\mathrm{C}), 90^{\circ}(\mathrm{S}), 50^{\circ}$ and $140^{\circ}(\mathrm{V})$, and $90^{\circ}(\mathrm{Y})$, respectively. We note that the minima tended to occur at the same orientations as the negative zero crossings of bias, a finding that will be explored in connection with Figure 11. We will now turn to an analysis of each of the effects noted above and will start with the relationship between bias and aspect ratio, while grouping the results over stimulus orientations.

Effect of aspect ratio on bias. In order to obtain insight into the effect of aspect ratio on bias, we pooled the data for each aspect ratio across orientations. Pooling was performed by taking the root mean square (RMS) of the biases for a given aspect ratio. RMS was calculated by first taking the square of the biases, calculating the mean (over all 12 orientations) of the squares, and then taking the square root of the mean. The RMS was used, rather than the mean, because biases were both positive and negative at different orientations and they tended to cancel each other out when averaged.

As aspect ratio increases (the ellipses become more elongated), the accuracy of observers' responses improves (RMS bias decreases). All the observers showed this pattern. We go beyond the qualitative statement that an increase in aspect ratio enhances performance by identifying a transformation of aspect ratio. The transformed variable is approximately linearly related to both the RMS bias and the circular standard deviations of the observers' settings. We term the transformed aspect ratio the roundness $(\boldsymbol{r})$ and define it by

$$
r=1 /(\alpha-1),
$$

where $\alpha$ is the aspect ratio. Thus, ellipses with a high roundness have a low aspect ratio and are more circular, and ellipses with a low roundness have a high aspect ratio and are more elongated.

We observe a linear relationship between RMS bias and roundness for all the observers, shown in Figure 4. This was confirmed by weighted linear regressions, using the inverse of the $95 \%$ confidence interval as weights. We note that the regression of RMS bias is somewhat ad hoc. Looking at the plots, it seems that the data of Observers $\mathrm{B}, \mathrm{C}, \mathrm{S}$, and $\mathrm{V}$ might be fit better with a compressive function. However, a compressive function would have more parameters, leading to problems of model comparison. Thus, we view the regressions of Figure 4 and Table 1 as descriptive, rather than as the best model to fit the data.

The results of these regressions are given in Table 1. Four out of six regressions are significant, and all slopes are positive. The slopes vary widely between observers, but all the results indicate an increase in RMS bias with increasing roundness. The intercepts differ significantly from zero for all the observers. The intercept corresponds to the RMS bias for an ellipse of zero roundness, which is an infinitely long line. The slope gives the increase in RMS bias with a unit increase in roundness.

Table 1

Results of a Weighted Linear Regression of Root Mean Square Bias on Roundness for Each Observer

\begin{tabular}{cccccccc}
\hline Observer & Intercept & $95 \%$ CI & Slope & $95 \% \mathrm{CI}$ & $R^{2}$ & $F$ & $p$ \\
\hline A & 0.48 & 0.08 & 0.40 & 0.10 & .650 & 9.3 & .02 \\
B & 0.10 & 0.08 & 0.37 & 0.09 & .622 & 11.5 & .006 \\
C & 0.18 & 0.11 & 0.88 & 0.18 & .836 & 25.5 & .002 \\
S & 0.13 & 0.06 & 0.68 & 0.16 & .906 & 28.9 & .01 \\
V & 0.39 & 0.23 & 0.58 & 0.31 & .305 & 2.2 & .21 \\
Y & 0.25 & 0.12 & 0.18 & 0.13 & .094 & 0.63 & .46 \\
\hline
\end{tabular}

Note-The intercepts and slopes and their $95 \%$ confidence intervals (CIs) are in degrees. The coefficient of determination is calculated from the correlation between the predicted root mean square bias and the observed ones. 

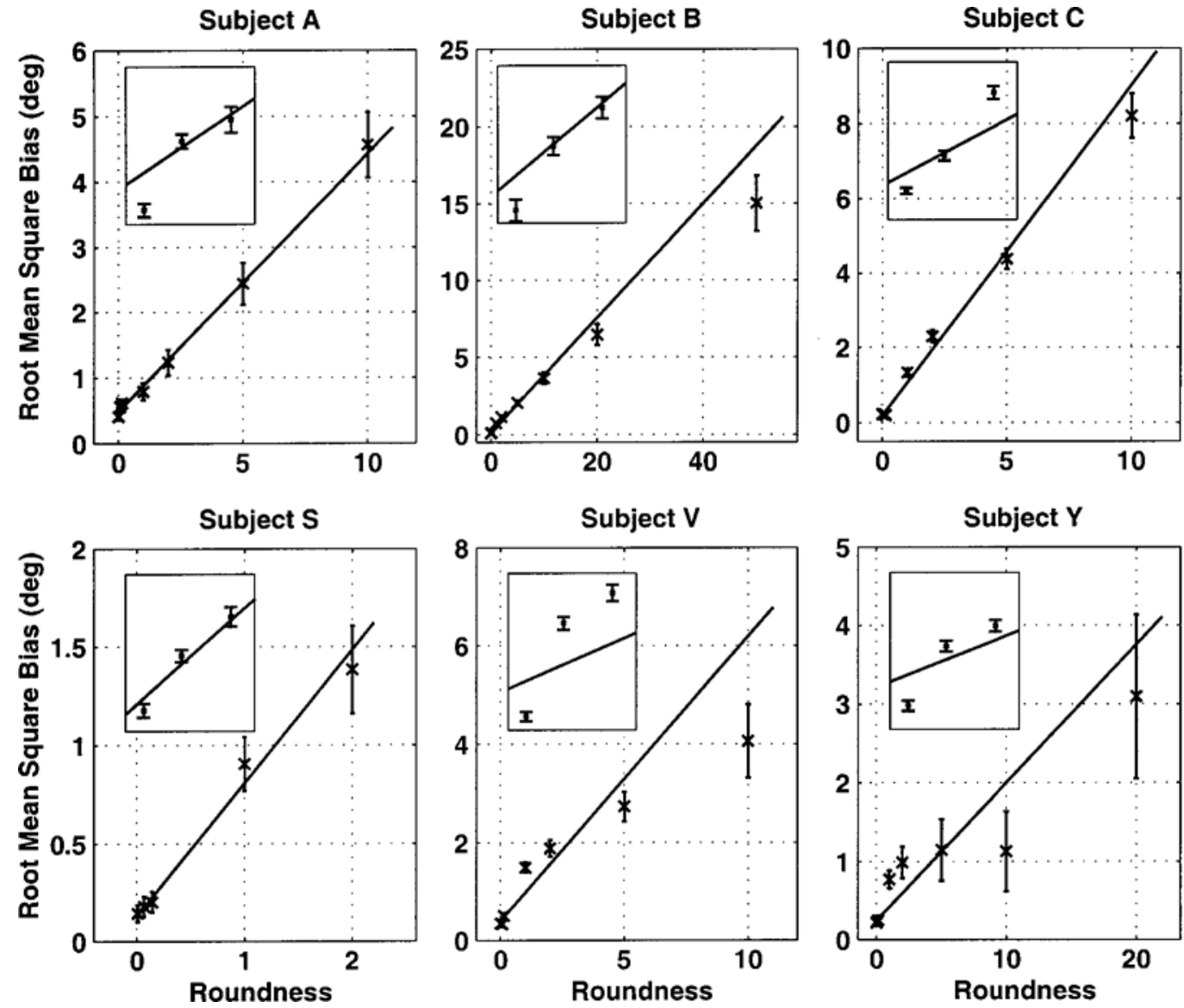

Figure 4. All the observers' root mean square biases (in degrees) as a function of roundness. The lines are the weighted least-squares regression fits for each observer. The small plots at the upper left corner in each plot are the enlargements of the three responses for a roundness less than 0.2 . The regression lines are not necessarily parallel to the large plot, because the range of the $x$-axis of the small plot is kept constant for all the subjects and the scale of the $y$-axis is adjusted for optimal viewing.

Effect of aspect ratio on circular standard deviation. Prior to analyzing the circular standard deviations as a function of roundness, we pooled the responses across orientations. Pooling was performed by subtracting the circular mean from each block of settings for a given ellipse orientation and aspect ratio and then grouping all the subtracted results across orientations for each aspect ratio. The circular standard deviation of the resulting sample was calculated, using the method described in Appendix B. The circular standard deviation decreases with increasing aspect ratio. By expressing the results as a function of roundness (see Figure 5), the circular standard deviations seem to lie on a straight line. As in the case of the RMS bias, the circular standard deviation is well fit by a linear regression on the roundness. As can be seen in Figure 5, the regression line falls within the $95 \%$ confidence interval for most of the data points for all 6 observers. The confidence interval was calculated using the bootstrap method described in Appendix B.

Note that because the confidence interval was not constant as a function of roundness (it increased with the circular standard deviation), an unweighted linear regression was not the most appropriate fitting method. Thus, we performed a weighted regression for the data in Figures 4 and 5, in which the weights were the widths of the confidence intervals. A drawback of this type of regression is that it contains no constant term; also, the interpretation of the coefficient of determination $\left(R^{2}\right)$ is problematic, because it can become larger than 1 in certain situations. As a way around this problem, we calculated $R^{2}$ as the Pearson correlation coefficient between the predicted circular standard deviations and the observed ones (the correlation between $y$ and $\hat{y}$; see Judge, Hill, Griffiths, Lutkepohl, \& Lee, 1988, p. 213). We used the $F$ test as a second measure of the quality of the fits (Draper \& Smith, 1998, pp. 38ff).

Table 2 contains the results of the regressions. First, all but one of the regressions are significant $(p<.05)$. Observer $\mathrm{C}$ does not show a significant linear relationship but seems to show a negatively accelerated relationship. The intercept is the precision (grouped over orientations) when the observer views an infinitely long line 

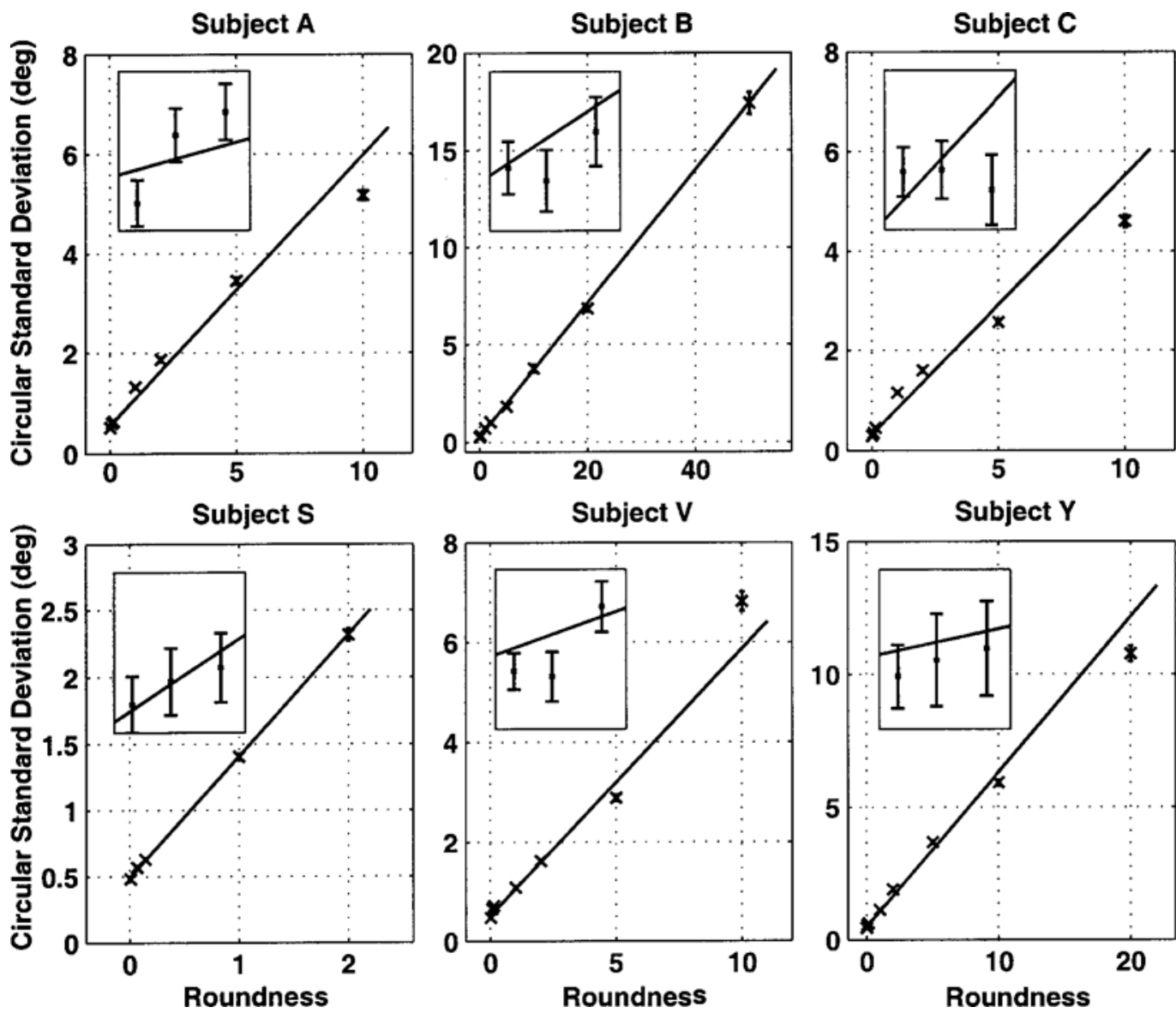

Figure 5. All the observers' circular standard deviations (in degrees) as a function of roundness. The lines are the weighted least-squares regression fits for each observer. The small plots at the upper left corner in each plot are the enlargements of the responses for a roundness less than $\mathbf{0 . 2}$. The regression lines are not necessarily parallel to the large plot, because the range of the $x$-axis of the small plot is kept constant for all the subjects and the scale of the $y$-axis is adjusted for optimal viewing.

(roundness $=0$ ). Thus, it is the fraction of the precision that is independent of the aspect ratio. The slope gives the increase in circular standard deviation for a unit increase in roundness.

Effect of orientation: Oblique effect. In order to determine whether our results exhibit the oblique effect, we compared the RMS biases and circular standard deviations across the oblique orientations (averages of $15^{\circ}, 30^{\circ}, 45^{\circ}$, $60^{\circ}, 75^{\circ}, 105^{\circ}, 120^{\circ}, 135^{\circ}, 150^{\circ}$, and $165^{\circ}$ ) with those for the cardinal orientations (averages of $0^{\circ}$ and $90^{\circ}$ ). We performed this analysis only for the larger aspect ratios; the results for aspect ratios less than 1.2 (roundness $>5$ ) were not included, because they are influenced by the observers' intrinsic preferences, as we will discuss in Experiment 2. One-sided $T$ tests were carried out to compare the differences in RMS bias and circular standard deviation between oblique and cardinal orientations for each aspect ratio. The statistical comparisons were all significant $(p<.05)$; the biases and circular standard deviations were significantly larger at oblique orientations than at the cardinal orientations. The results corroborated the oblique effect.
In Figure 6, we plot the cardinal/oblique ratio for the RMS bias and the circular standard deviation for each observer as a function of roundness. We chose to plot these data against roundness, instead of aspect ratio, because the conditions would be more evenly spaced. A ratio smaller than 1 indicates that the RMS bias and circular standard deviation are smaller in the cardinal than

Table 2

Results of a Weighted Linear Regression of Circular Standard Deviation on Roundness for Each Observer

\begin{tabular}{cccccccc}
\hline Observer & Intercept & 95\% CI & Slope & $95 \%$ CI & $R^{2}$ & \multicolumn{1}{c}{$F$} & $p$ \\
\hline A & 0.56 & 0.10 & 0.56 & 0.12 & .762 & 16.0 & .007 \\
B & 0.27 & 0.03 & 0.34 & 0.03 & .920 & 80.5 & $<.0001$ \\
C & 0.33 & 0.07 & 0.52 & 0.17 & .455 & 4.7 & .085 \\
S & 0.49 & 0.03 & 0.92 & 0.08 & .979 & 140.0 & .001 \\
V & 0.56 & 0.10 & 0.51 & 0.14 & .668 & 10.0 & .02 \\
Y & 0.50 & 0.07 & 0.58 & 0.08 & .774 & 20.5 & .002 \\
\hline
\end{tabular}

Note-The intercepts and slopes and their $95 \%$ confidence intervals (CIs) are in degrees. The coefficient of determination is calculated from the correlation between the predicted circular standard deviations and the observed ones. 

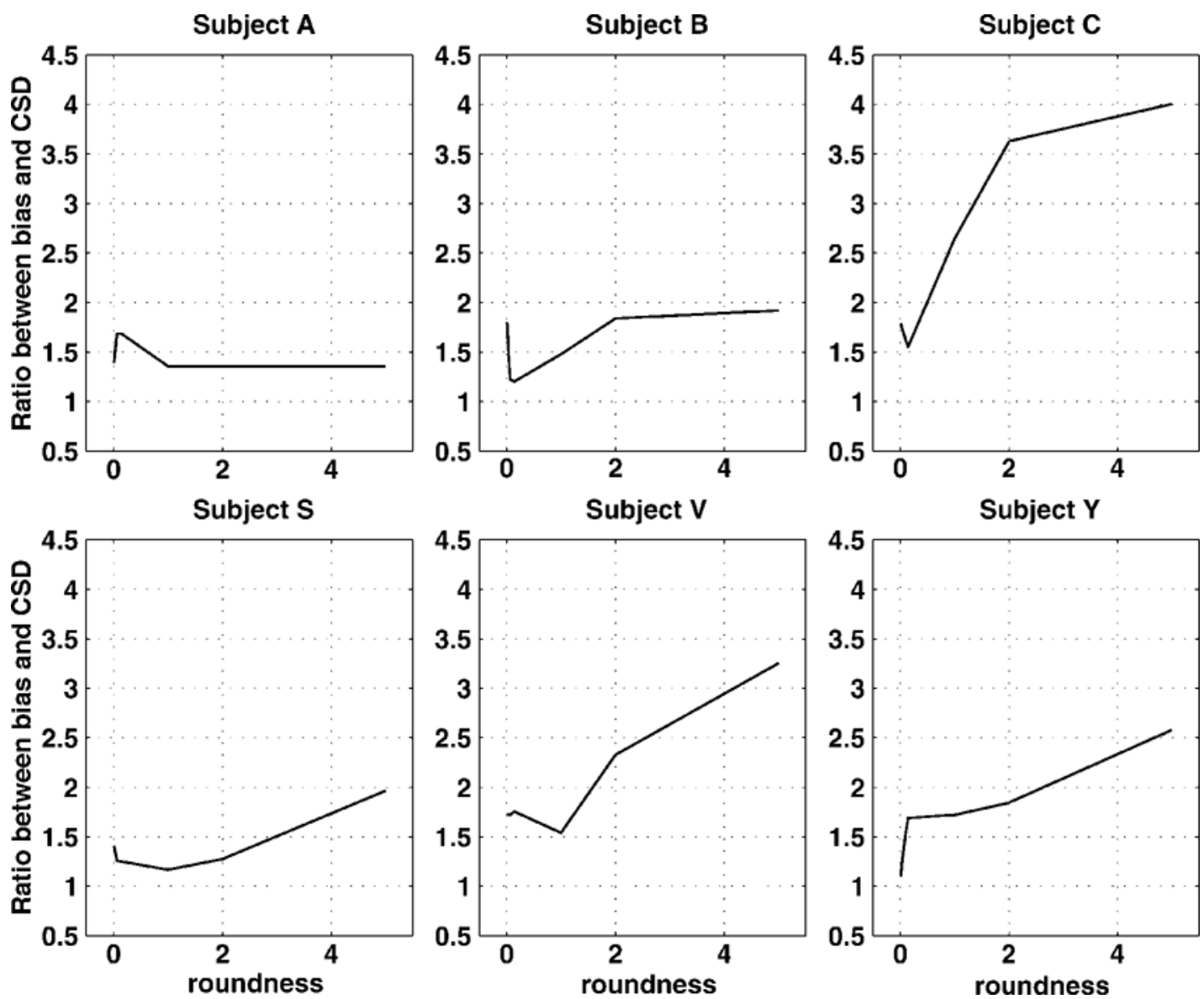

Figure 6. The ratio between biases and circular standard deviations (CSDs) under cardinal and oblique orientations as a function of roundness for all the observers. The circles are the ratio for circular standard deviation; the crosses are the ratio for biases. Only roundness less than or equal to 5 (i.e., aspect ratio larger than or equal to 1.2) is shown. The error bars for the ratio between circular standard deviations are the $95 \%$ confidence interval, and the horizontal bar is the mean of the ratios for circular standard deviation.

in the oblique orientations; in other words, it indicates that observers' judgments of orientation are more accurate and precise at cardinal orientations. All of the ratios are less than 1, indicating that all the observers exhibited the oblique effect in every condition. Also shown in Figure 6 is the mean circular standard deviation over all values of roundness for each subject (horizontal line). With few exceptions, this mean lies within the $95 \%$ confidence interval for each of the circular standard deviations. Thus, we can conclude that the cardinal/oblique ratio of circular standard deviation is independent of roundness. A weighted linear regression of the cardinal/ oblique ratio of the circular standard deviation on roundness supports the hypothesis that the cardinal/oblique ratio is independent of roundness: The regression is not significant for all the subjects $(p>.1)$, except Subject $Y$ $(p=.03)$. The size of the ratio ranges from .78(Observer $\mathrm{S}$ ) to .44 (Observer $\mathrm{V}$ ), and the average ratio over all observers is .57 .
As was stated above, the cardinal/oblique ratio of RMS bias is always smaller than one, indicating the oblique effect. We did not plot the confidence intervals for this ratio in Figure 6, since the error bars are often larger than 1, thus covering the whole vertical extent of the plot. This is due to the nature of the bias, since the bias at the cardinal directions is often not significantly different from zero, which leads to large confidence intervals in the cardinal/oblique ratio.

Individual differences and reliability. The results plotted in Figures 4, 5, and 6 show that individual observers differed considerably from each other. But are these results reliable? One way to bring out the internal consistency is through test-retest correlations. We divided the data into even- and odd-numbered trials, calculated bias and circular standard deviation for each half of the data, and then correlated the results for the two halves. For the between-subjects correlation, we lumped together the biases and circular standard deviations for 
each observer in two 108-element $(9$ aspect ratios $\times 12$ orientations) arrays and correlated these arrays between each pair of subjects. However, in doing so, we found that the correlations were dominated by the results from the low aspect ratios, since the bias and circular standard deviations are larger for low aspect ratios than for high ones. Therefore, we normalized the biases for each aspect ratio condition with the pooled RMS biases (plotted in Figure 4) and the circular standard deviations with the pooled circular standard deviations (plotted in Figure 5). This way, each condition contributes roughly equally to the final Pearson correlation coefficient. In Figure 7, we show the test-retest correlations between all pairs of observers both for bias (left panel) and for circular standard deviation (right panel).

As one can see, the test-retest correlations of an observer with him- or herself (circles) are considerably higher than the between-observers correlations (crosses). This is especially true for the bias and less so for the circular standard deviation. We wondered about the relatively low test-retest correlations of an observer with him- or herself for the circular standard deviations. We believe these relatively low test-retest correlations are caused by chance fluctuations, since the sample size of 40 is relatively small and more samples are generally required to estimate a variance than to estimate a mean. To back this belief up, we ran a Monte Carlo simulation, repeatedly estimating the standard deviation for data drawn from a normal distribution (linear statistics). We found a similar distribution of test-retest correlations between standard deviation estimates with a sample size of 40, which indicated that the small sample size caused the low testretest correlations in variance.

Lastly, we performed two test-retest analyses in which we sliced the data up in a different way. In one analysis, we took the first half and second half of the trials. This led to slightly lower within-subjects correlations than those shown in Figure 7. This indicates small adaptation effects for all the subjects. The second way was by dividing the trials in a 1-2,3-4 pattern (in every group of four data points, the first two data go to one group, and the second two go to another group). This led to results similar to those shown in Figure 7, indicating that the results do not depend on the way the data is divided in halves.

\section{Discussion}

Our results replicated the oblique effect: The orientation of ellipses at cardinal orientations was seen with higher accuracy and precision than at oblique orientations. This is consistent with numerous results reported in the literature (Andrews, 1967; Appelle, 1972; Bouma \& Andriessen, 1968; Howard, 1982; Li \& Westheimer, 1997; Vogels \& Orban, 1985; Westheimer \& Beard, 1998). This is no surprise, but it nicely establishes the consistency of our results with earlier experiments despite the different shapes that we used.

We find an effect of aspect ratio on orientation perception. Observers are more accurate and more precise for high aspect ratio stimuli than for low aspect ratio ones. By expressing the aspect ratio as a new variable, we established linear relationships between roundness and performance. We found a linear relation between roundness and the RMS of the biases. In the case of precision, the relationship between grouped circular standard deviation and roundness is also well fit by a line, although there is a suggestion of a compressive relationship for high values of roundness. A possible explanation of the linear relationship between precision and roundness is provided in the General Discussion section.

The fact that we find a linear relationship between performance and roundness does not necessarily mean that roundness itself is the variable that explains performance. It could be a variable that covaries with roundness. We discern three plausible variables that covary with roundness: (1) the ease with which the extreme points (the points where the long axis connects to the
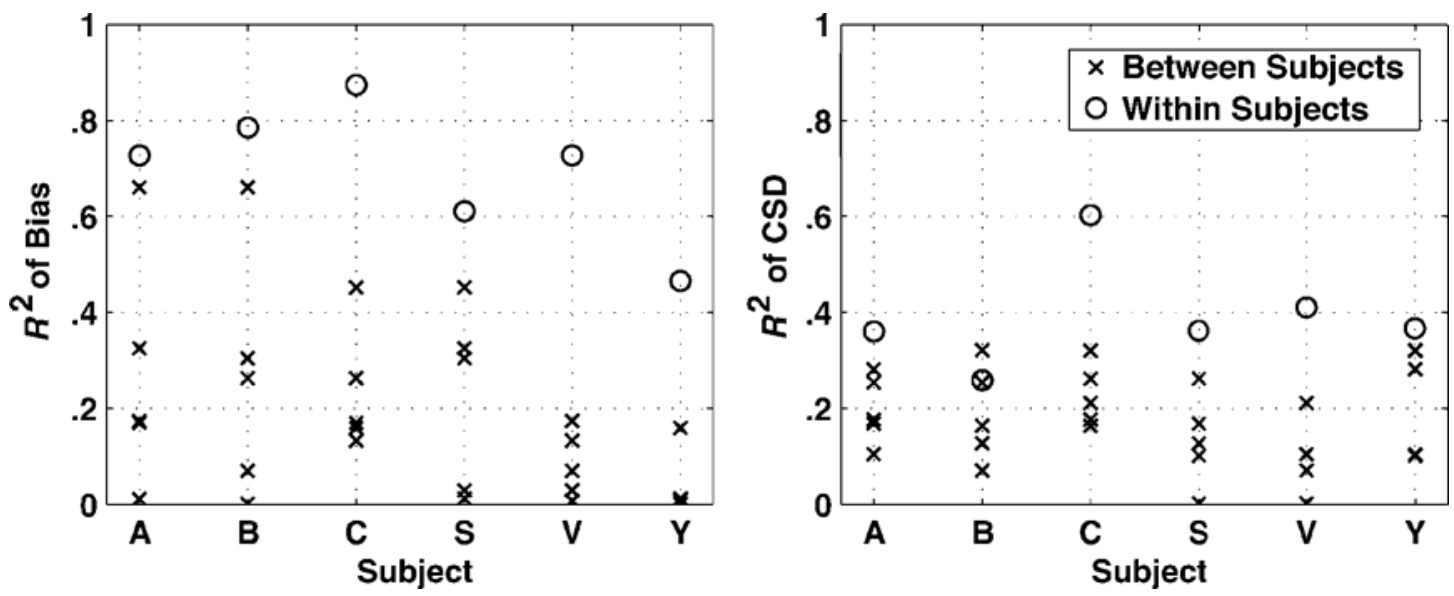

Figure 7. Between-subjects and within-subjects (test-retest) correlations (squares of the correlation coefficients) plotted for each observer. Left panel, bias; right panel, circular standard deviation. 


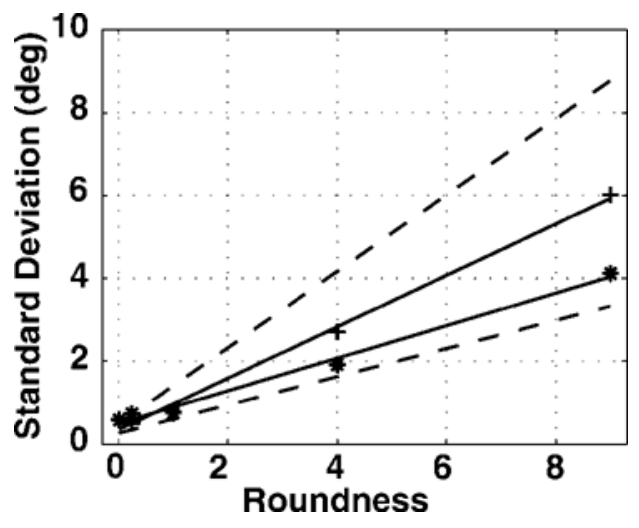

Figure 8. A replot of the data of Figures $1 \mathrm{a}$ and $\mathrm{Lb}$ in $\mathrm{Li}$ and Westheimer (1997). The vertical axis is the circular standard deviation (in degrees) calculated from their discrimination thresholds; the horizontal axis is the roundness calculated from their aspect ratios. The "+" symbol is for $L i$ and Westheimer's Subject E.L.; the "*" symbol is for their Subject W.L. The two solid lines denote a least-squares fit to their data. The dashed curves show the regression lines of Subjects $B$ and $S$ in our Experiment 1.

edge of the ellipse) can be located, (2) the length of the long axis, and (3) the area of the ellipse. The extreme point is needed for the strategy of distance minimization, in which the subject minimizes the distance between the extreme point and an inside endpoint of one of the probe line segments. We will discuss the possible contribution of the distance minimization strategy below, in connection with Li and Westheimer's (1997) data. As for the possibility that the length of the long axis or the ellipse area explains performance, we can make use of a design constraint in the experiment. Recall that we kept area constant for aspect ratios smaller than 2 and that we kept the length of the long axis constant for aspect ratios larger than and equal to 2 . Thus, the slopes of the lines describing performance as a function of roundness should be different under these two regimes if either of these two variables affects performance. We compared separate regressions of performance on roundness for roundnesses larger than 1 (i.e., an aspect ratio of 2) and for roundnesses less than or equal to 1 . We found only one significant slope difference between the two regimes (Subject S, for circular standard deviation), making the length of the long axis and the ellipse area unlikely explanatory variables. Therefore, aspect ratio per se affects observers' performance in our experiment. Aspect ratio has also been shown to be the variable that explains performance in the discrimination of rectangles of different shapes (Regan \& Hamstra, 1992). By randomizing the size, length, and width of the rectangles, Regan and Hamstra were able to show that aspect ratio is the explanatory variable, excluding such alternatives as size and length of short and long axes.

As was mentioned above, Li and Westheimer (1997) performed a study quite similar to ours. We have replot- ted their data in Figure 8 to determine whether or not the two sets of results are consistent. We transformed their $75 \%$ correct discrimination thresholds to standard deviations (effectively, $86 \%$ thresholds) by assuming that the underlying psychometric function has the form of a cumulative normal distribution function. Thus, we multiplied Li and Westheimer's discrimination thresholds by a factor of 1.48 . We then regressed the resulting standard deviations on roundness as described above; the result of this regression is plotted for their 2 observers as solid lines. The dashed lines are the 2 extreme observers (Observers B and S) in our experiment. The range of behaviors exhibited by our observers encompasses that of $\mathrm{Li}$ and Westheimer's observers.

Comparison with Li and Westheimer's (1997) data brings up two points. First, Li and Westheimer used the method of constant stimuli. Since we used the method of adjustment to collect data, the similar findings suggest that the results do not depend on the psychophysical method used for data collection. Second, Li and Westheimer used a one-interval forced-choice paradigm in which subjects were asked to judge whether the long axis of an ellipse was clockwise or counterclockwise from the vertical. Thus, their subjects could not use a strategy based on minimization of the distance between extreme points of the ellipse and inside points of the probe line. Since the results of the 2 observers of $\mathrm{Li}$ and Westheimer fall squarely within the range observed in our data, it is unlikely that the minimization strategy is a major factor in our results.

The last main finding in Experiment 1 is that the pattern of biases and circular standard deviations is consistent within observers, but not across observers. Although the subjects were reliable, there were substantial individual differences. In order to explore these individual differences in more depth, we designed Experiment 2.

\section{EXPERIMENT 2 \\ Intrinsic Preferences From Circle Settings}

The results from Experiment 1 showed that there were considerable individual differences in observers' performance of adjusting orientations. In this experiment, we added a circle (aspect ratio of 1) to the set of stimuli in order to measure the intrinsic orientation preference of our observers in the absence of any orientation information in the stimulus. In order to mask the presence of the circle, we included other ellipses with small aspect ratios.

\section{Method}

Design. This was a $5 \times 12$ within-subjects design. Aspect ratio $(1.0,1.05,1.2,2.0$, and 4.0$)$ and orientation $\left(0^{\circ}, 15^{\circ}, 30^{\circ}, 45^{\circ}, 60^{\circ}\right.$, $75^{\circ}, 90^{\circ}, 105^{\circ}, 120^{\circ}, 135^{\circ}, 150^{\circ}$, and $165^{\circ}$ ) were the two independent variables.

Stimuli and Procedure. The stimuli were ellipses (including circles), as described in the General Method section. There was no practice for this session. The observers were not told that there were circles among the stimuli. The 6 observers did 10 blocks of trials during a period of 1 week. In each block, each combination of the 
Table 3

Pearson Correlation Coefficients for Each Observer Between the Results of Experiment 1 and Experiment 2

\begin{tabular}{lllllll}
\hline & \multicolumn{6}{c}{ Observer } \\
\cline { 2 - 7 } \multicolumn{1}{c}{ Correlation } & $\mathrm{A}$ & $\mathrm{B}$ & $\mathrm{C}$ & $\mathrm{S}$ & $\mathrm{V}$ & $\mathrm{Y}$ \\
\hline Biases & .91 & .65 & .94 & .74 & .61 & .63 \\
Circular standard deviations & .83 & .95 & .96 & .57 & .87 & .97 \\
\hline
\end{tabular}

Note-All the correlation coefficients are significant $(p<.01)$, except for Observer S's correlation coefficient of circular standard deviation $(p>.05)$.

12 orientations and five aspect ratios was presented twice randomly (120 trials). For each observer, there were 20 repeats for each condition, and the total number of trials for each observer was 1,200.

\section{Results}

The observers did not complain about this experiment when the circle condition was mixed in among the stimuli, and they did not find the task impossible to do. The possible reason is that they considered the circles as ellipses of very low aspect ratio, whose orientation was just difficult to perceive. One response was removed from the data set $(1 / 7,200=0.01 \%)$, using the same considerations as in Experiment 1.

Comparison with results of Experiment 1. Because we pool the results from Experiments 1 and 2 in some of the analyses, it is important to determine whether or not the observers behaved similarly in both experiments. Some of the conditions in this experiment were identical to those in Experiment 1 (aspect ratios of 1.05, 1.2, and 2.0 ); we performed $T$ tests to compare the two sets of biases and circular standard deviations. We excluded conditions that yielded circular standard deviations greater than $15^{\circ}$. Most of the bias results $(95 \%)$ and the standard deviations (97.5\%) from Experiment 2 were not significantly different in those from Experiment $1(p>.05)$. The correlations between the results of Experiment 1 and Experiment 2 are all significant, except for 1 subject $(p<.01$; listed in Table 3$)$.

The effects of orientation and aspect ratio were similar to those in Experiment 1. The bias and circular standard deviation were smaller around the vertical and the horizontal than at other orientations. For aspect ratios greater than or equal to 1.2, $T$ tests were carried out separately for each observer between data for cardinal and for oblique orientations. For biases, 17 out of the 18 comparisons were significant $(p<.05)$. Most of the comparisons $(94.4 \%, 17$ out of 18$)$ between circular standard deviations were significant $(p<.05)$. As in Experiment 1 , the relationships between RMS bias, circular standard deviation, and roundness are well described by a straight line.

Distribution of orientation responses to the circle. Figure 9 shows the distributions of the settings of all the observers when the stimulus was a circle. The observers showed very distinctive patterns, but none of them showed a uniform distribution of settings. A uniform distribution might occur if subjects simply left the probe at its initial (random) orientation. Observers S and $\mathrm{Y}$ had a clear preference for $90^{\circ}$ (vertical), although Observer Y's distribution was much broader. Observers $\mathrm{C}$ and $\mathrm{V}$ had roughly bimodal distributions, with $\mathrm{C}$ preferring both $55^{\circ}$ and $130^{\circ}$ and $\mathrm{V}$ preferring $60^{\circ}$ and $140^{\circ}$. Observer $\mathrm{C}$ preferred $130^{\circ}$ much more strongly than $55^{\circ}$, whereas Observer V preferred both orientations roughly equally. Observer A showed a relatively narrow peak around $75^{\circ}$, and $\mathrm{Ob}-$ server B had a broad peak around $50^{\circ}$ but also showed responses for most other orientations. Interestingly, our observers showed little intrinsic preference for the horizontal. Lastly, we performed an analysis to see whether the subjects changed their circle settings distribution during the experiment. Such a change might explain the bimodal distributions of Subjects C and V. The analysis was performed visually by plotting all the circle setting data as a function of block number. Although crude, visual inspection showed no obvious change of distribution with block number, strengthening our belief that these distributions reflected an intrinsic preference.

Influence of intrinsic preference. Figure 10 shows the distribution of responses of Observers $\mathrm{A}$ and $\mathrm{V}$ for the circle and their biases and circular standard deviations for an aspect ratio of 1.05 . The peaks in the response frequency distribution for the circle are close to the negative zero crossings of the bias and to the minima of the circular standard deviation. Observer A had a preference for orientations around $75^{\circ}$. His bias pattern shows a negative zero crossing around $75^{\circ}$, and his circular standard deviation is minimal around that orientation. We call this overlap data pattern the line-up pattern.

Observer V had preferences for orientations around $60^{\circ}$ and $140^{\circ}$. The preferences for $60^{\circ}$ and $140^{\circ}$ appear as dips in the circular standard deviation profile, whereas they appear as negative zero crossings in the bias pattern. Other observers have different negative zero crossings of bias and minima of circular standard deviation, but the pattern is the same as those for Observers $\mathrm{A}$ and $\mathrm{V}$ : The peaks of the distributions of circle settings line up with the negative zero crossings of the bias and the minima of the circular standard deviations. The line-up pattern for all the observers is shown in Figure 11.

In Figure 11, we show the zero crossings of the bias (plus and minus symbols), the minima of the circular standard deviation (circles), and the means and circular standard deviations of the distributions of circle settings (gray area). These three quantities were calculated from the pooled data of Experiments 1 and 2, using the following procedure. For each roundness, the zero crossings of bias were calculated by considering the bias as a function of orientation. We compared each consecutive pair of bias values and checked whether the line connecting them crossed zero. If it did, we report the orientation in which the line crossed zero as a zero crossing. In order to avoid spurious zero crossings, the biases were low-pass filtered with a Butterworth filter with a cutoff frequency of 0.5 (where 1 is the Nyquist frequency). In order to avoid clutter, only the negative zero crossings 

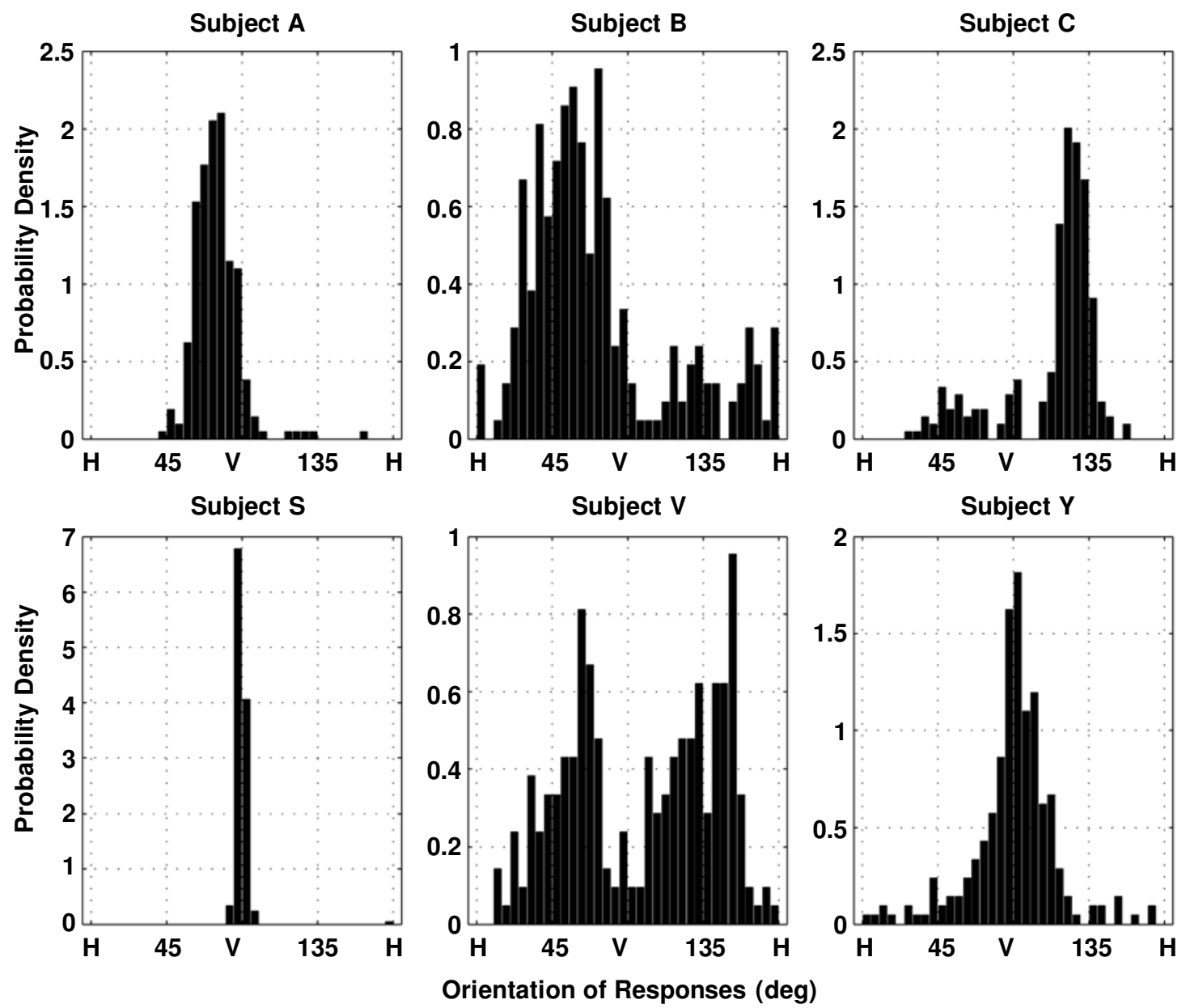

Figure 9. Probability density histograms of the responses of each observer under the circle condition. The bin size of the histogram is $5^{\circ}$.

are plotted in Figure 11 for the higher roundnesses and only the positive ones for the lower roundnesses. The boundaries between high and low roundness are 10,50, $10,2,10$, and 20 for the 6 observers, respectively. The zero crossings that are not plotted in Figure 11 show no clear structure.

The circular standard deviations were identically filtered. To find a minimum, we considered five consecutive circular standard deviation values and stipulated that the middle one be lower than the other four. If this was the case, we fitted a quadratic function through the five points and used the minimum of the fit as the minimum of the circular standard deviation. The location and width of the circle settings were calculated as the mean and circular standard deviation of the distributions (Appendix B). For the observers with bimodal distributions (Observers $\mathrm{C}$ and $\mathrm{V}$ ), we calculated the mean and circular standard deviation for each half of the data separately (one half comprising all settings smaller than $90^{\circ}$, the other half for all settings larger than $90^{\circ}$ ).

As Figure 11 illustrates, the observers' intrinsic preferences tended to influence their responses at high values of roundness (at which there was less stimulus information about orientation); the oblique effect dominated the results at lower roundnesses. With the gray box, we purport to show an influence zone of the intrinsic preferences found in the circle settings. Within the gray box, biases and circular standard deviations show the line-up pattern. The vertical extent of the gray box was calculated by the mean orientation plus and minus one circular standard deviation of the circle settings. The horizontal extent was calculated from the top of the linear region (to be discussed below) to the maximal roundness value used (50). The only exception for the setting of the boundary was Observer B, for whom we set, somewhat arbitrarily, the lower horizontal bound of the gray box to a roundness of 35 . As one can see, most of the negative bias zero crossings fall in or are very close to the gray box. Two exceptions came from Observer C, with roundnesses of 50 and 20. For this observer, there were clear "kinks" in the relationship between bias and ellipse orientation, but these kinks were not big enough to cross zero (data not shown). The kinks occurred around an orientation of $60^{\circ}$ and, thus, would have fallen in the gray 
Subject A
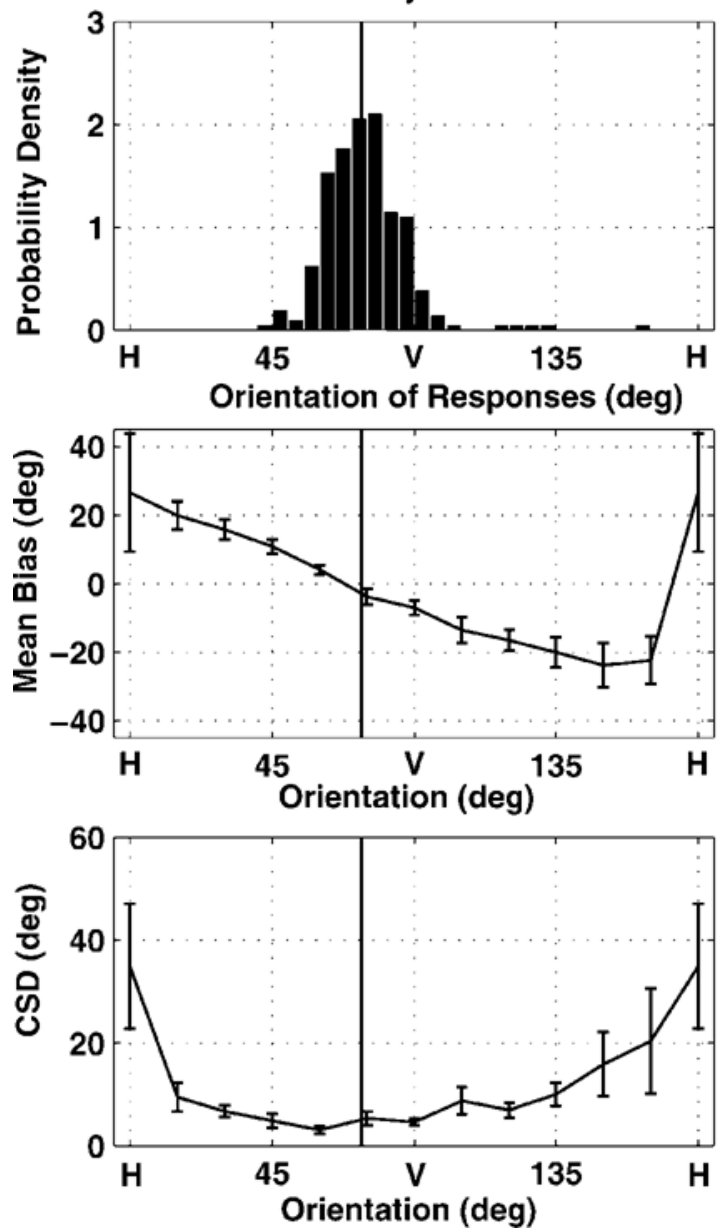

Subject $V$
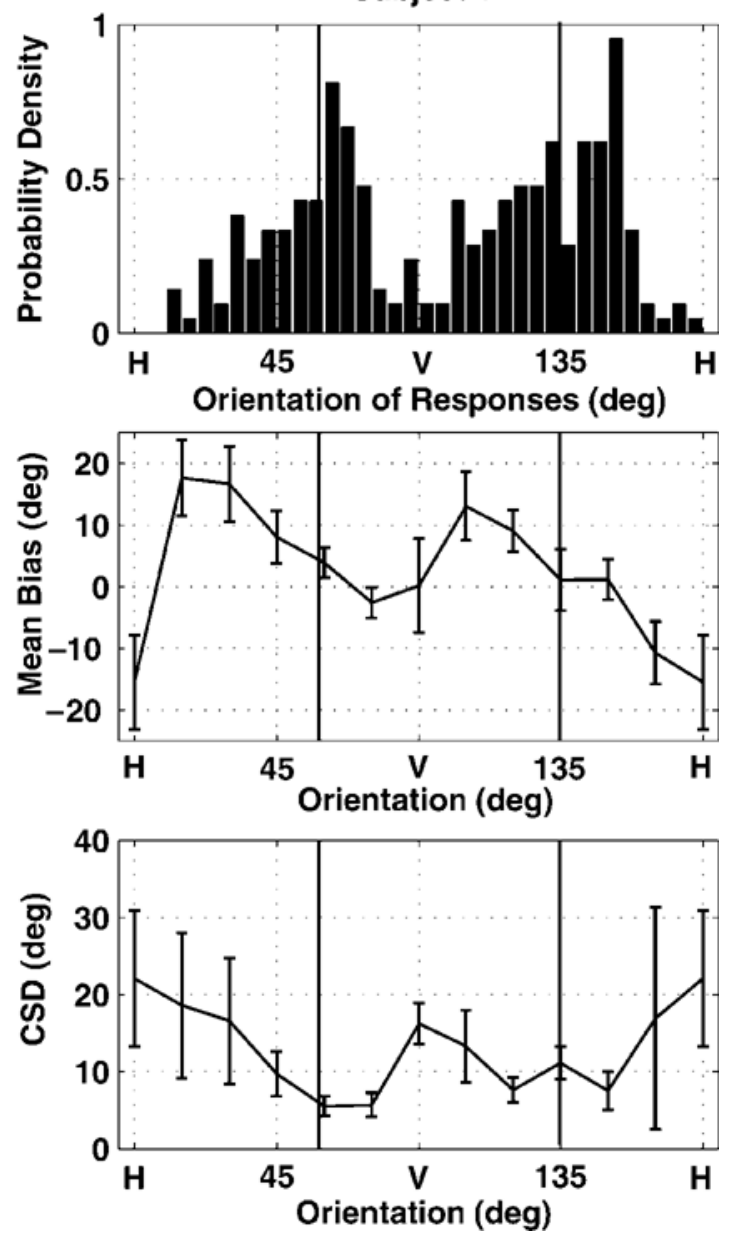

Figure 10. Observer A's and Observer V's probability density histograms of responses under the circle condition (top panels), their bias (middle panels), and their circular standard deviation (CSD, bottom panels) as a function of the orientation of stimuli for an aspect ratio of 1.05. The vertical lines are around the orientations at which the observers responded most frequently under the circle condition. The negative zero crossings in the bias pattern and the minima of the circular standard deviations are close to the most frequently responded orientations under the circle condition.

area had the bias crossed zero. Furthermore, all but one minimum of circular standard deviation fall in or are close to the gray box. The lone exception is Observer $\mathrm{S}$, with a roundness of 50 . We have no explanation for this single anomalous case. For the lower roundnesses, we observe that the positive zero crossings of bias and the minima of circular standard deviation tend to fall at the principal orientations. This is another way of illustrating the oblique effect.

The line-up pattern described above persists for the high roundnesses. We call the range of roundnesses that show the line-up pattern the line-up regime. For the higher aspect ratios, the line-up pattern is lost in favor of a pattern like the ones shown in Figure 3 for aspect ratios of 1.5 and 16. The threshold aspect ratio, below which the line-up pattern is found, depends on the width (expressed as a circular standard deviation) of the circle settings distribution. More specifically, the aspect ratio threshold depends on a comparison of the width of the circle settings' distribution and the circular standard deviation of conditions with an aspect ratio larger than 1 . This is plotted in Figure 12.

In Figure 12, we show the circular standard deviation as a function of roundness for all the observers, using the data of Experiment 1. The plots show the full range of roundness, in contrast to Figure 5, where we showed only one part of the aspect ratios. In addition, we show the width of the intrinsic preferences distribution plotted as a horizontal line. As can be seen for all but 1 observer (B), the horizontal line intersects the circular standard deviation as a function of roundness. We think of the horizontal line as specifying an influence threshold for the intrinsic preference. Aspect ratio conditions with a circular standard deviation close to or above the horizontal line are influenced by the intrinsic preference; those below are not. This naturally leads to a division of 

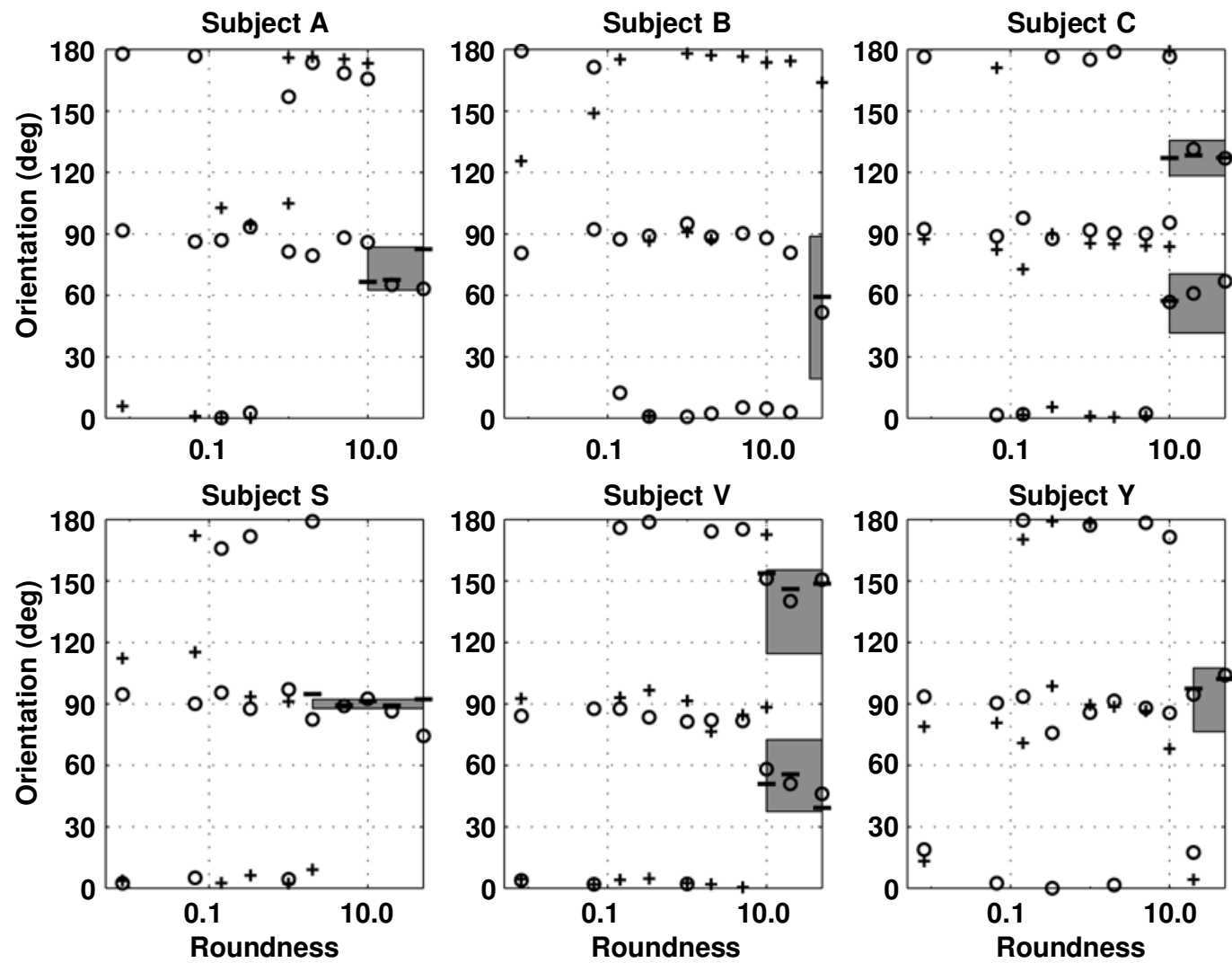

Figure 11. Positive (+ symbol) and negative (- symbol) zero crossings and minima of circular standard deviations $(\bigcirc$ symbol) for each subject and each roundness condition. The gray boxes denote the influence zones of the circle settings. The vertical extent of the gray boxes is determined by the mean plus and minus one circular standard deviation, and the horizontal extent is determined by the linear regime (see the text).

the aspect ratio conditions into two regimes. The regime above the threshold roundness is the line-up regime. The regime below and close to the threshold roundness is the linear regime, where the relationship between circular standard deviation and roundness is linear. The roundness thresholds are 10,50,10,2,10, and 20 for Subjects $\mathrm{A}, \mathrm{B}, \mathrm{C}, \mathrm{S}, \mathrm{V}$, and $\mathrm{Y}$, respectively. To test that this regime is indeed a linear one, we redid the regression of circular standard deviation on roundness and added the next largest roundness condition to the regression, as compared with the regression in Figure 5. For Observer B, it was not possible to add an extra roundness condition, since all of his data had already been used in the regression of Figure 5. Consistent with our labeling of the regime as linear, we found a reduced $R^{2}$ in these regressions for all the other subjects.

\section{Discussion}

The results in Experiment 2 replicated the results from Experiment 1 and reinforced the conclusion that the performance of orientation perception of ellipses is reliable. The oblique effect and the effect of linearly increasing error with increasing roundness were also found. The distribution of responses in the circle condition that were shown in Figure 9 can be considered as a glimpse into the observers' minds. With no information about orientation available in the stimulus, the only thing the observers relied on was their intrinsic preference for certain orientations. The more peaked this distribution was (i.e., the less the width of the distribution), the more the responses to noncircular ellipses were influenced by the intrinsic preference. In particular, the observer (S) with the narrowest intrinsic preference had the largest line-up regime, the 4 observers (A, C, V, and Y) with a medium width of intrinsic preference had a medium-sized line-up regime, and the observer (B) with the broadest intrinsic preference had the smallest line-up regime. Inversely, the less peaked the distribution for the intrinsic preferences was, the larger the linear regime, where the percept was dominated by the ellipse stimulus. Thus, the responses seem to have been the result of a balance between the information from the world and the observers' preferences in their minds.

For the higher aspect ratios, we find that accuracy and precision are higher in the principal orientations than in the oblique ones. These results replicate the oblique ef- 

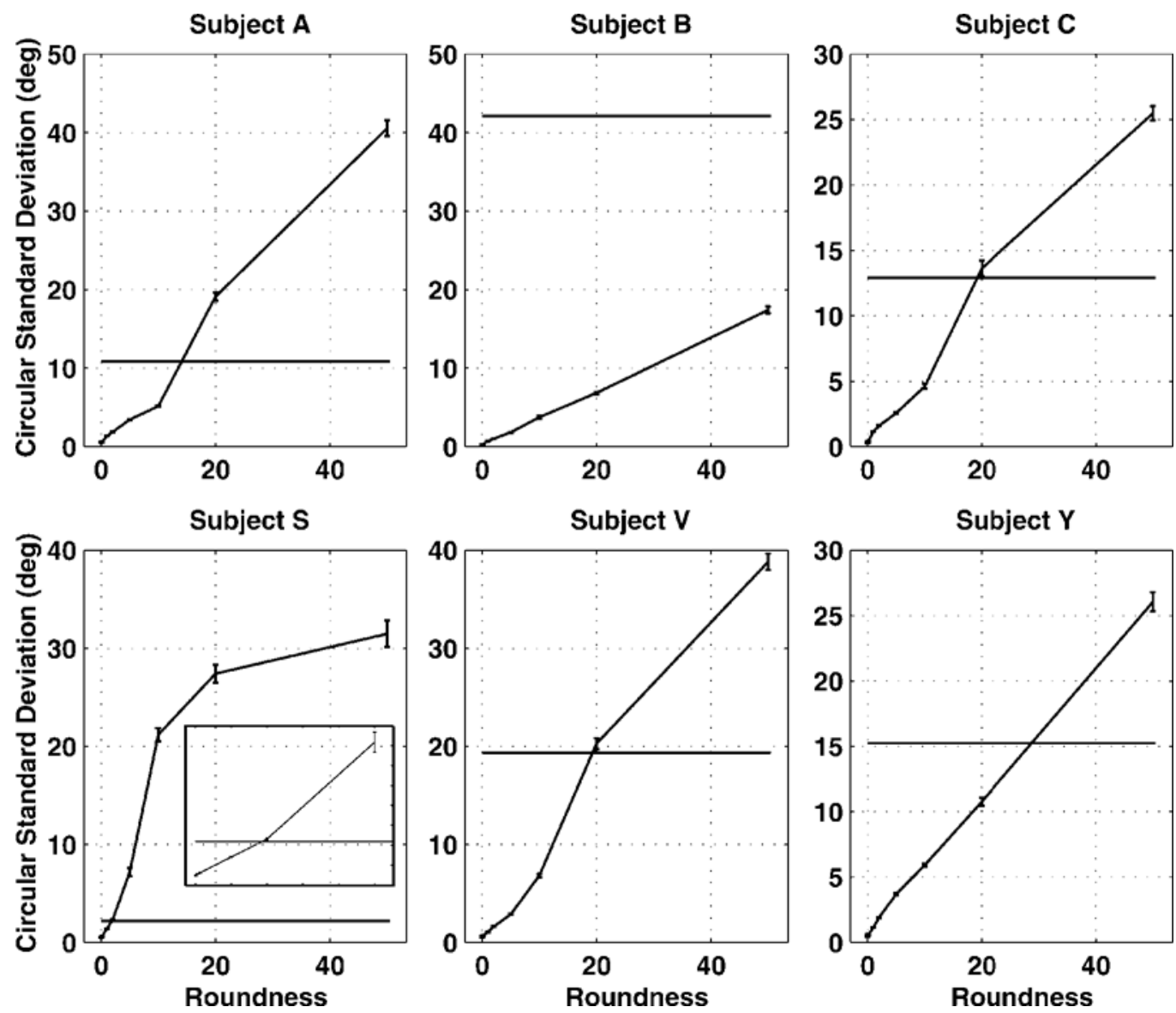

Figure 12. Circular standard deviations for each subject as a function of roundness. The full range of roundness is used here, unlike in Figure 5. The horizontal lines indicate the circular standard deviations of the probability density histograms of Figure 9. For Subject $S$, the inserted figure is an enlargement of the data points under the lowest four roundness conditions.

fect. For the accuracy, this means that the bias is zero. There are two ways this could potentially occur: positive and negative zero crossings. We find almost exclusively positive zero crossings at the cardinal orientations for the higher aspect ratios, which indicates a repulsion from the vertical or horizontal orientation. This differs from Greene, Frawley, and Swimm (2000) and Andrews (1967), who reported a variety of bias patterns.

\section{EXPERIMENT 3 Noisy Ellipse Orientation Perception}

The results of the first two experiments showed the oblique effect for the orientation perception of ellipses, demonstrated that increasing aspect ratio leads to higher performance, and showed that individual differences in intrinsic preference influence the response to the low aspect ratio ellipses. To investigate whether these results also hold for more generic shapes, we randomly perturbed the contour of ellipses to make the shape irregular.

\section{Method}

Stimuli. We generated irregular ellipses by adding low-pass filtered, normally distributed white noise to the boundary of regular ellipses. Each noise sample was an array of 120 elements, 1 for each vertex of the polygon making up the ellipse. The noise had a standard deviation of $7 \mathrm{~mm}$ and was convolved with a Gaussian with a standard deviation of four vertices. The noisy ellipses were constructed by adding a sample of the noise to each of the $x$ and $y$ coordinates (effectively doubling the noise power) of a 120 -sided elliptical polygon. The polygon was generated as described in the Method section of Experiment 1. The stimulus set consisted of 12 orientations $\left(0^{\circ}, 15^{\circ}, 30^{\circ}, 45^{\circ}, 60^{\circ}, 75^{\circ}, 90^{\circ}, 105^{\circ}, 120^{\circ}, 135^{\circ}, 150^{\circ}\right.$, and $\left.165^{\circ}\right)$, five aspect ratios $(1.0,1.2,1.5,2.0$, and 4.0), and 20 noise samples for each of the orientation and aspect ratio conditions. The same set of 1,200 irregular ellipses was presented to each observer (see Figure 13 for a few examples of the stimuli).

Calculation of aspect ratio and orientation. The aspect ratio and orientation of the perturbed ellipses do not necessarily coincide with those of the unperturbed one. Thus, we used the second-order central moment matrix to calculate the aspect ratio and orientation of the shape (Horn, 1986, section 3.2). We calculated the aspect ratio from the ratio of the eigenvalues and the orientation from the eigenvector of the major axis. Even though the aspect ratios and the 


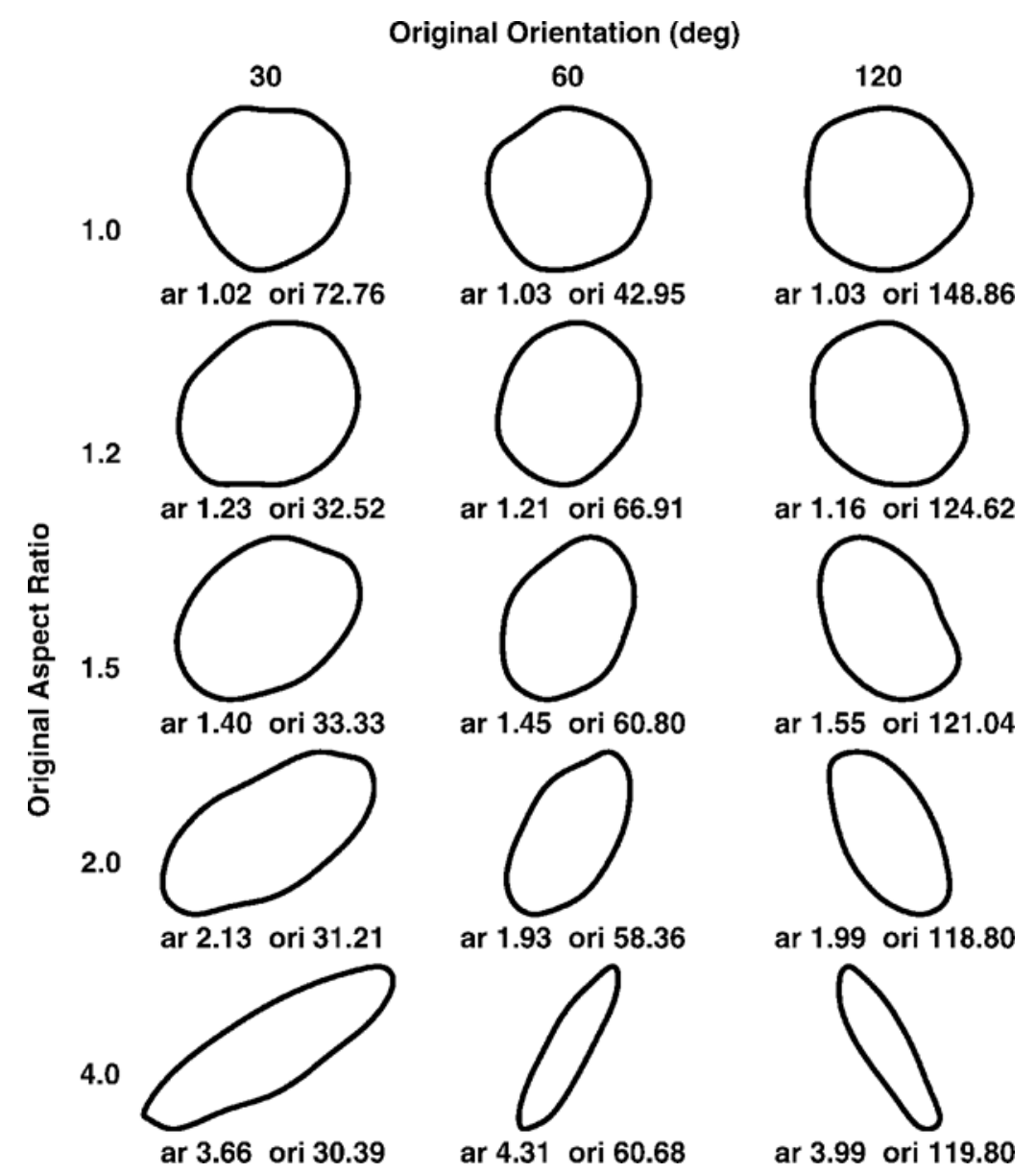

Figure 13. A sample of ellipses with randomly perturbed boundaries with different aspect ratios and orientations. The original aspect ratio and orientation of the ellipses that the stimuli were based on are listed at the top of each column and on the left of each row. For stimuli with an aspect ratio of 1.0, the orientation does not have a meaning. The actual fitted aspect ratio and orientation obtained from the second-order moments are indicated below each ellipse.

orientations of the irregular ellipses were random, they were clustered around the original values of the ellipses that they were based on. We grouped the aspect ratios and orientations. Aspect ratios were grouped around $1.02(1.01-1.1), 1.2(1.1-1.3), 1.5(1.3-1.7)$, $2.0(1.7-2.3)$, and $4(3.5-4.5)$. The orientations were similarly grouped into $15^{\circ}$ bins. Twenty-eight shapes had aspect ratios outside of the specified ranges; these shapes were dropped from the analyses of the results.

Design. This experiment used a within-subjects design. The aspect ratio and orientation were the two independent factors. The stimuli were grouped into five different aspect ratios: 1.02, 1.20, 1.50, 2.0, and 4.0. The orientations of the irregular ellipses were grouped into 12 settings: $0^{\circ}, 15^{\circ}, 30^{\circ}, 45^{\circ}, 60^{\circ}, 75^{\circ}, 90^{\circ}, 105^{\circ}, 120^{\circ}, 135^{\circ}, 150^{\circ}$, and $165^{\circ}$.

Procedure. Each observer did 10 blocks of trials during a period of 2 weeks. In each block, 120 irregular shapes were presented.

\section{Results}

Eight responses were excluded from the analysis $(0.12 \%)$, using the same criteria as those in Experiments 1 and 2 .

Biases and circular standard deviations. The observers' biases were two to three times larger than those in Experiments 1 and 2, and the circular standard deviations were three to five times greater. Observer A's bias and circular standard deviation results are shown in Figure 14. Observer A's biases were about twice as large as those in Experiment 1, and circular standard deviations were three to five times as large as in the corresponding conditions in Experiment 1. Other observers behaved similarly. The biases were less around the vertical and the horizontal, although this trend is not as clear as in the first two experiments. In the comparison between cardinal and oblique orientations for aspect ratios larger than or equal to $1.2, T$ test results showed that $71 \%$ of the comparisons between oblique and cardinal orientations were significant $(p<.05)$.

In addition, the relationship between bias and aspect ratio and that between circular standard deviation and aspect ratio are similar to those in Experiments 1 and 2: As aspect ratio increases, the bias and circular standard deviation decreases. We quantified this relationship by a linear regression of the RMS of biases and circular stan- 

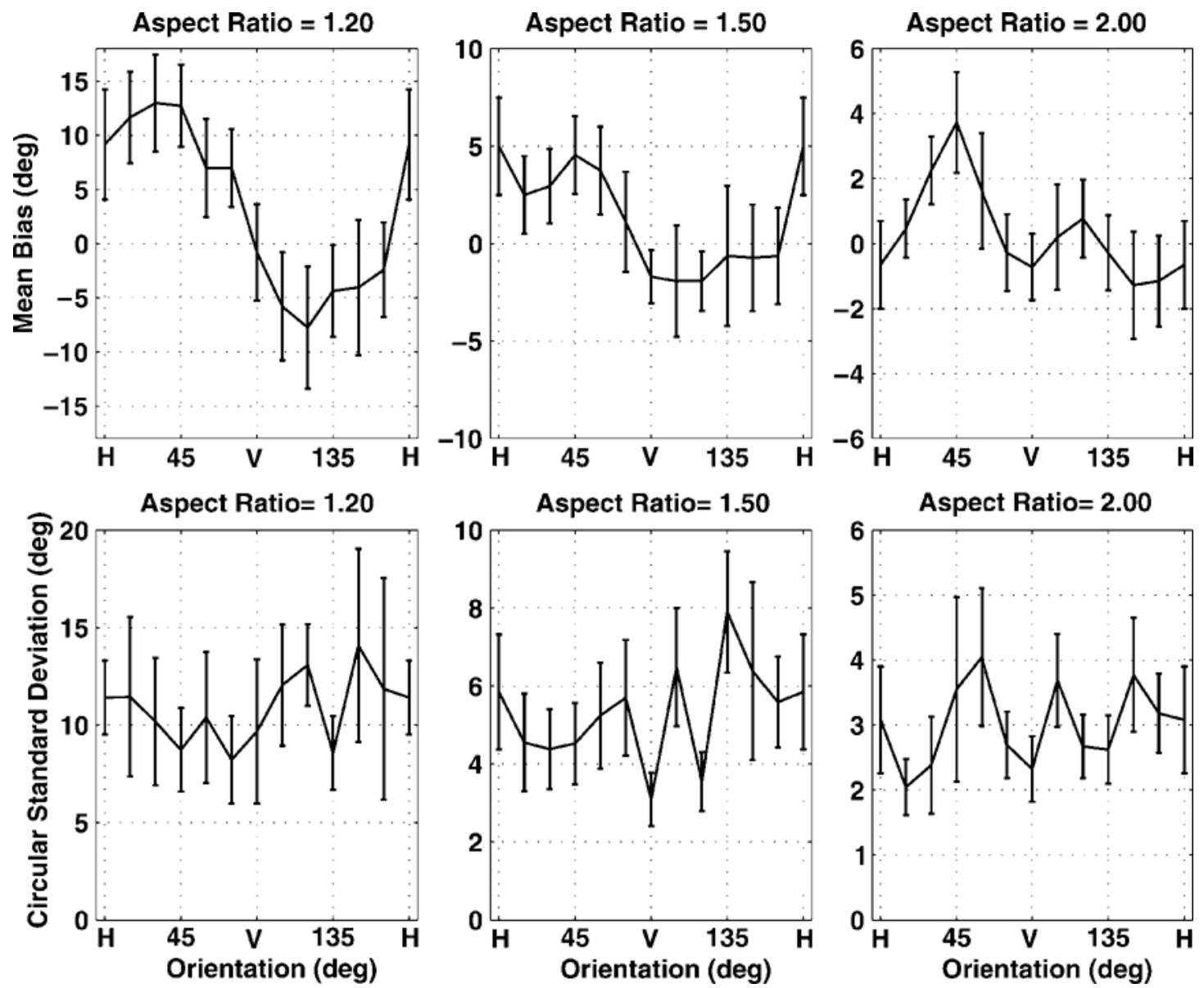

Figure 14. Observer A's results for aspect ratios of 1.2, 1.5, and 2.0. The top row shows the bias results; the bottom row shows the circular standard deviation results. The error bars denote the $95 \%$ confidence interval.

dard deviation on roundness, similar to the ones plotted in Figures 4 and 5. All the regressions had $R^{2}>.9$ in all cases. As a measure of how much performance suffered from the noise we added to the ellipse, we calculated the ratios of the regression slopes for each subject between this experiment and Experiment 1. The bias slope increased by a factor of 1.5-3.5 (overall mean ratio, 2.8), and the circular standard deviation slope increased by a ratio of 3.5-5.5 (overall mean ratio, 4.4).

Distribution of responses. Despite the lower accuracy and precision, each observer's responses showed a consistent pattern. With the exception of Observer V, the distribution of each observer's responses for aspect ratios 1.01-1.1 (see Figure 15) showed a preference, for certain orientations, similar to that found in the circle condition in Experiment 2 (Figure 9). Observer V did not show a clear preference in Experiment 3, but only a bias against orientations around the horizontal.

\section{Discussion}

The lower accuracy and precision found in Experiment 3, as compared with Experiments 1 and 2, indicate that the orientation of irregular shapes is more difficult to perceive than that of ellipses. However, even though the task became more challenging, the results still showed the oblique effect and the influence of aspect ratio. Moreover, the pattern of biases was similar to that observed in the earlier experiments, and the observers' intrinsic preferences in Figure 15 are similar to those in Figure 9. The results demonstrate that individual preferences not only play a role in orientation perception of ellipses, but also affect the orientation perception of irregular shapes.

\section{GENERAL DISCUSSION}

We investigated the visual perception of 2-D shape orientation. As stimuli, we used simple shapes with either a perfect elliptical contour or a randomly perturbed elliptical contour. They were chosen from a set of shapes with a wide range of aspect ratios: from a circle to a straight line. The shapes were presented in the fronto-parallel plane in 12 orientations $\left(0^{\circ}, 15^{\circ}, \ldots, 165^{\circ}\right)$, and the task of the observers was to set a probe line in the same orientation as the shape. The probe was a broken line sur- 

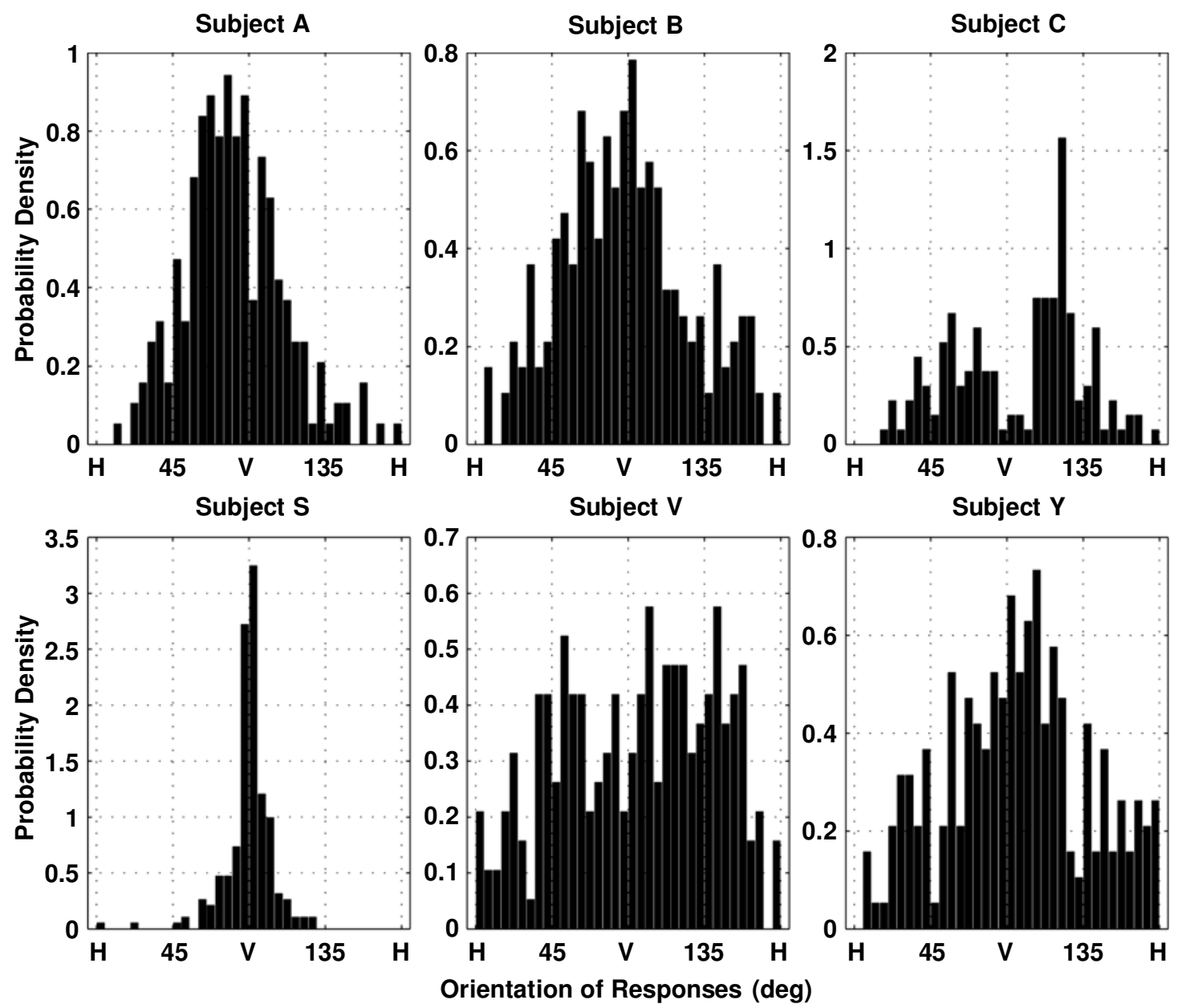

Figure 15. Probability density histograms of the responses of each observer for aspect ratios from 1.01 to 1.1. The bin size of the histogram is $5^{\circ}$.

rounding the stimulus, and it could be rotated around the common centroid of the ellipse and the probe line.

The results are expressed in terms of accuracy (bias, the difference between the circular mean and the orientation of the long axis of the ellipse) and precision (variability, the circular standard deviation around the mean) of the settings. Performance improves with an increasing aspect ratio of the shape: Both the biases and the standard deviations become smaller when the shape is more elongated. Inversely, a rounder shape provides less information about its orientation and leads to a decrease in performance. We defined a new variable called roundness, which is $1 /($ aspect ratio -1$)$, and found linear relationships between roundness and performance.

The results of Experiment 1 show substantial individual differences, and to gain more insight into this phenomenon, we added the circle condition in Experiment 2. This revealed the intrinsically preferred orientations of the observers, since the stimulus itself did not have an orientation. It turned out that the orientation distributions showed idiosyncratic patterns for different observers and could explain the performance in the conditions with elon- gated shapes. More specifically, we showed that the peaks in the distribution of circle settings occurred at the same orientations as the negative zero crossings of bias and the minima of the circular standard deviations. What could underlie these intrinsic preferences? We cannot answer this question unequivocally with the present data. However, we can exclude a few simple explanations. First, since we checked for astigmatism, we can exclude it as a possible cause. Second, it is unlikely that a deterministic squashing function (an astigmatism of the brain) can explain the results. With such a squashing function, it is hard to explain the bimodal intrinsic preferences of Observers $\mathrm{C}$ and $\mathrm{V}$. Also, the squashing function would predict that an ellipse with its long axis orthogonal to the squashing axis should appear as a circle and, thus, the subjects should show a uniform distribution of responses to this ellipse. Since we never found a uniform distribution of settings in any of our conditions, the squashing function explanation is unlikely.

According to the effect of intrinsic preferences and the relationship between performance and roundness, we distinguish two categories of shapes for orientation perception in our experiments. 
The first category is the line-up regime. For shapes with low aspect ratios (high values of roundness), the intrinsic preference of the observer dominates the performance. The orientation of the shape is so weakly conveyed by the stimulus that the observer's intrinsic preferences dominate the percept. This category is characterized by a range of low aspect ratios that starts at 1 and, depending on the observer, ends between 1.02 and 1.5. For these aspect ratios, we find a line-up of intrinsic preferences with negative zero crossings of bias and minima of circular standard deviation.

The second category is the linear regime. The higher aspect ratio shapes show an increasing performance with an increasing aspect ratio. The accuracy increases linearly if bias is plotted as the pooled RMS bias as a function of roundness. The precision also increases linearly if the circular standard deviation is plotted against the roundness. Orientation is perceived better when the shape looks more elongated and the corresponding aspect ratios range from a range (1.02 to 1.5 for different observers) to line.

We replicated the well-known oblique effect: The cardinal orientations $\left(0^{\circ}\right.$ and $\left.90^{\circ}\right)$ lead to higher accuracy and precision than do other orientations. Although our results do not allow us to draw any direct conclusions about the possible causes of the oblique effect, there is one aspect of our data that indirectly sheds some light on this issue. The discussion about the origin of the oblique effect has often been framed in terms of the properties of pools of neurons (Howard, 1982; Schoups, Vogels, Qian, $\&$ Orban, 2001). We found that the oblique effect is different or, sometimes, even absent under low aspect ratio conditions. This finding is hard to reconcile with the properties of a single fixed pool of neurons. Why would the neurons change their properties when the stimulus is weak? Although our results certainly do not argue against a neural explanation, they indicate that more is needed than a model based on a single fixed pool of neurons. Below, we will briefly describe a modeling approach that is complementary to a neural one.

A detailed model exceeds the scope of the present paper. However, we want to briefly describe our favored approach, which is Bayesian in nature (see Knill \& Richards, 1996). A Bayesian model consists of two components: a prior probability distribution, which captures the observer's prior knowledge concerning the set of possible stimuli, and a likelihood function that quantifies the unbiased probability of a stimulus. In our approach, the intrinsic preference (circle settings) corresponds to the Bayesian prior probability distribution, and a function derived from an ideal observer model for the ellipse takes the place of the Bayesian (stimulus) likelihood. In the ideal observer model, an ellipse is represented as a polygon (just as is the stimulus on the screen in the experimental display). Normally distributed noise is added to each vertex of the polygon. The major axis of the polygon is determined by fitting an ellipse to the noisy polygon. The fitting is performed for many independent noise samples in a Monte Carlo fashion. Plotting the circular standard deviation of the fitted major axis angle versus roundness of the ellipse results in a linear relationship, which signifies the aptness of the transformation of aspect ratio to roundness. The stimulus likelihood as derived from the ideal observer model is unbiased and independent of ellipse orientation (i.e., it peaks at the true ellipse orientation). The Bayesian posterior probability distribution is computed by multiplication of the prior probability distribution and the likelihood function. In the model, the posterior distribution calculated in this manner is equivalent to the distribution of the observer's response settings. Thus, model predictions for the bias and circular standard deviation can be derived from the posterior distribution and compared with actual data.

In our experiments, significant individual differences were found. The patterns of intrinsic preference were idiosyncratic, and the between-subjects correlations were lower than the within-subjects correlations. Individual differences in the context of orientation perception have been observed before. Greene et al. (2000) measured the ability to position a point collinear with a line segment that was presented in different orientations. They found that the profile of biases was different for different observers but that observers showed the same profile when tested on different days. They found test-retest correlations larger than .60, and these are comparable to the results in our experiments. Koenderink, van Doorn, and Kappers (1992) studied the perception of surface shape from photographs of objects by using the gauge probe method. Observers had to adjust an attitude probe consisting of a small disk and a perpendicular stick to indicate the local orientation of the surface. By combining measurements at different positions in the photograph, Koenderink et al. reconstructed the 3-D surface and used these reconstructions to determine between- and within-subjects correlations. The analyses showed high within-subjects correlations and low between-subjects correlations.

One major source of the individual difference comes from intrinsic preference, and one way to think of intrinsic preference is as the beholder's share of the percept, as introduced by Gombrich (1959) in the context of the perception of art. The stimulus itself does not "cause" the percept in any deterministic way. The percept is the result of the interaction of the observer with the (optical) world. Here, we introduced a method to explicitly measure the beholder's share by having subjects judge a neutral stimulus condition-in this study, the orientation of a circle. This allowed us to look at individual differences in their purest form.

\section{REFERENCES}

ANDREws, D. P. (1967). Perception of contour orientation in the central fovea: Part I. Short lines. Vision Research, 7, 975-997.

Appelle, S. (1972). Perception and discrimination as a function of stimulus orientation: The "oblique effect" in man and animals. Psychological Bulletin, 78, 266-278.

Bouma, H., \& Andriessen, J. J. (1968). Perceived orientation of isolated line segments. Vision Research, 8, 493-507. 
Bowker, D. O., \& Mandler, M. B. (1981). Apparent contrast of suprathreshold gratings varies with stimulus orientation. Perception \& Psychophysics, 29, 585-588

Coppola, D. M., Purves, H. R., McCoy, A. N., \& Purves, D. (1998). The distribution of oriented contours in the real world. Proceedings of the National Academy of Sciences, 95, 4002-4006.

DrAPER, N. R., \& SMITH, H. (1998). Applied regression analysis. New York: Wiley.

FISHER, N. J. (1993). Statistical analysis of circular data. Cambridge: Cambridge University Press.

Gombrich, E. H. (1959). Art and illusion. London: Phaidon.

Greene, E., Frawley, W., \& SWimm, R. (2000). Individual differences in collinearity judgment as a function of angular position. Perception \& Psychophysics, 62, 1440-1458.

Heeley, D. W., \& Buchanan-Smith, H. M. (1990). Recognition of stimulus orientation. Vision Research, 30, 1429-1437.

Horn, B. K. P. (1986). Robot vision. Cambridge, MA: MIT Press.

Howard, I. P. (1982). Human visual orientation. New York: Wiley.

Judge, G. G., Hill, R. C., Griffiths, W. E., Lut kepohl, H., \& Lee, T. (1988). Introduction to the theory and practice of econometrics. New York: Wiley.

KnILl, D. C., \& Richards, W. (1996). Perception as Bayesian inference. New York: Cambridge University Press.

Koenderink, J. J., van Doorn, A. J., \& KAPPers, A. M. L. (1992). Surface perception in pictures. Perception \& Psychophysics, 52, 487-496.
Li, W., \& Westheimer, G. (1997). Human discrimination of the implicit orientation of simple symmetrical patterns. Vision Research, 37, 565-572.

Maloney, L. T., \& KoH, K. (1988). A method for calibrating the spatial coordinates of a visual display to high accuracy. Behavior Research Methods, Instruments, \& Computers, 20, 372-389.

Oomes, A. H. J., \& DiJkstra, T. M. H. (2002). Object pose: Perceiving 3-D shape as sticks and slabs. Perception \& Psychophysics, 64, 507520.

REGAN, D. (2000). Human perception of objects. Sunderland, MA: Sinauer Associates.

Regan, D., \& Hamstra, S. J. (1992). Shape discrimination and the judgement of perfect symmetry: Dissociation of shape from size. $\mathrm{Vi}$ sion Research, 32, 1845-1864.

Schoups, A., Vogels, R, Qian, N., \& Orban, G. (2001). Practising orientation identification improves orientation coding in V1 neurons. Nature, 412, 549-553.

Switkes, E., Mayer, M. J., \& Sloan, J. A. (1978). Spatial frequency analysis of the visual environment: Anisotropy and the carpentered environment hypothesis. Vision Research, 18, 1393-1399.

Vogels, R., \& Orban, G. A. (1985). The effect of practice on the oblique effect in line orientation judgments. Vision Research, 25, 1679-1687.

Westheimer, G., \& Beard, B. L. (1998). Orientation dependency for foveal line stimuli. Vision Research, 38, 1097-1103.

\section{APPENDIX A \\ Spatial Precision of Stimulus and Probe Placement}

We will provide a rather detailed account of the precision of stimulus placement. We feel such a discussion is warranted, since the experimentally observed precision of settings of the most precise subject is very close to the precision of stimulus placement in our set-up. With the possible exception of a few conditions for these subjects, we can be sure that the observed precisions are not due to an artifact of stimulus placement.

The stimuli were presented using a frame-buffer. Thus, the stimuli were sampled, and the accuracy with which stimuli could be presented was limited by the size of the pixels. Furthermore, a cathode ray tube might introduce spatial deformations of the frame-buffer geometry (Maloney \& Koh, 1988). As we will show below, both of these effects were unlikely to have played a role in our set-up.

The highest precision that we found (over all observers and conditions) was a standard deviation of $0.13^{\circ}$. For 2 observers, we found several standard deviations below $0.3^{\circ}$; for the other 4 , they were always above $0.3^{\circ}$. The highest precision was always found in a horizontal or a vertical orientation (cardinal orientations); precision in an oblique orientation was usually worse by a factor of two. From geometrical considerations, it follows that accuracy of stimulus placement is best for cardinal orientations and worst for diagonal ones: The variation is a factor of the square root of 2 (about 1.4), which is the ratio of the length of the diagonal to the length or width of a square pixel. This factor of 1.4 was compared with the observed precision of the settings of the subjects. For all but 1 subject, we find that the precision of the settings for a diagonal ellipse was a factor of 1.5 or more, as compared with the precision of the settings for a horizontal or a vertical ellipse. The single exception was Subject Y, for whom we found a ratio of 1.05. However, Y's precision for the diagonal was so low $\left(0.5^{\circ}\right)$ that precision of stim- ulus placement was not a bottleneck. Thus, stimulus placement was most critical for the cardinal orientations.

There are three issues that need to be considered with respect to the use of a frame-buffer: placement accuracy of the ellipse, of the probe line, and of the mouse. Although it is possible to establish the accuracy with which the ellipse could be placed from first principles, we used an experimental approach. We drew one ellipse and then drew a second one that was rotated relative to the first one in increments of $0.01^{\circ}$. The second ellipse was drawn with an XOR mask, and thus, when there were any pixels that were not common to both ellipses, they would clearly show up. We repeated this procedure for a variety of aspect ratios and orientations and found the worst-case minimum angle to be around $0.05^{\circ}$. Since the best precision is considerably worse than $0.05^{\circ}$, we deem the frame buffer to be an insignificant factor.

The placement precision of the probe line can be calculated as follows. Each pixel is $350 / 1280=280 / 1024=0.273 \mathrm{~mm}$. Since the far end of the probe line is at a distance of $135 \mathrm{~mm}$ from the center, the worst-case angular resolution is $0.273 / 135=$ 0.00200 radians, which equals $0.116^{\circ}$. The SGI computer reports mouse coordinates in pixels. Since we instructed the observers to grab the far edge of the probe line, placement precision of the mouse is the same as that for the probe line. Thus, the worst precision of stimulus placement is $0.12^{\circ}$, which is close to the responses of 2 subjects from our experiments in some of the conditions (cardinal orientations and high aspect ratios) and might have influenced their precision. A further concern is the spatial deformation of the cathode ray tube in the monitor. One way to deal with this would be to measure the spatial deformation and correct for it (Maloney \& Koh, 1988). However, given that we wanted to use the method of adjustment in which observers are able to adjust the probe line in real time, 


\section{APPENDIX A (Continued)}

this was not an option, since the calculations to correct for the spatial deformation would slow down the computer too much. Instead, we performed a control experiment in which we rotated the monitor by $90^{\circ}$. We reasoned that if there was any significant distortion influencing the results, the results should change by rotating the monitor. We tested all the observers on a subset of the full conditions (for aspect ratios of $1.05,1.5$, and 16) and found no significant differences in accuracy and precision between the rotated and the upright monitor conditions. From this result, we concluded that spatial deformations did not significantly influence our results. In summary, it was unlikely that there is a significant influence of sampling by the pixel grid on the observers' performance. Furthermore, the influence of spatial deformations was negligible.

\section{APPENDIX B \\ Axial Circular Statistics}

Equation numbers refer to Fisher (1993), but note that the equations are adjusted for axial data.

Let $\theta_{i}$ be a random sample of size $n$ of axial angles $\left(0<\theta_{i}<\right.$ 180). Calculate the following intermediate quantities:

$$
\begin{aligned}
C= & (1 / n) \Sigma \cos \left(2 * \theta_{i}\right) \\
S= & (1 / n) \Sigma \sin \left(2 * \theta_{i}\right) \\
& R^{2}=C^{2}+S^{2} .
\end{aligned}
$$

The circular mean (Fisher Equations 2.7 and 2.9) is

$$
\bar{\theta}= \begin{cases}\frac{1}{2} * \tan ^{-1}(S / C) & S>0, C>0 \\ \frac{1}{2} *\left[\tan ^{-1}(S / C)+\pi\right] & C<0 \\ \frac{1}{2} *\left[\tan ^{-1}(S / C)+2 \pi\right] & S<0, C>0 .\end{cases}
$$

The circular standard deviation (Fisher Equation 2.12)

$$
v=1 / 2 *\{-2 \log (R)\}^{1 / 2} \text {. }
$$

In order to compare different circular means or circular standard deviations, one needs an estimate of their variability. As an estimate of the variability of the circular mean, we use the circular standard error; as an estimate of the variability of the circular standard deviation, we use the standard deviation derived from a bootstrap procedure.
The circular standard error (Fisher Equations 2.27, 2.28, 4.21, and 4.22) is calculated from:

the second central trigonometric moment,

$$
m=(1 / n) \sum \cos \left[2 *\left(2 * \theta_{i}-2 * \theta\right)\right],
$$

and the sample circular dispersion for the calculation of standard error,

$$
\delta=(1-m) /\left(2 * R^{2}\right) .
$$

The circular standard error is given by

$$
\sigma=1 / 2 *(\delta / n)^{1 / 2} .
$$

A $95 \%$ confidence interval for the circular mean is given by

$$
[\theta-\arcsin (1.96 \sigma), \theta+\arcsin (1.96 \sigma)] .
$$

A $95 \%$ confidence interval for the circular standard deviation (Fisher Equations 8.2 and 8.3) is calculated using balanced resampling. In this technique, many (typically 1,000 ) copies of the data are put in a big vector. Then a random permutation is used to shuffle the data. The shuffled data are parceled up into 1,000 surrogate data sets, and the circular standard deviation is calculated for each of them, leading to a distribution of circular standard deviations. The $95 \%$ confidence interval is obtained as the 97.5 th percentile of the distribution of circular standard deviations. 